Supporting Information for

\title{
Octahedral Iron Complexes of Pyrazine(diimine) Pincers: Ligand Electronic Effects and Protonation
}

\author{
Jaylan R. Billups, Zoe N. Fokakis, Sidney E. Creutz* \\ Department of Chemistry, Mississippi State University, Starkville MS 39762, United States \\ *screutz@chemistry.msstate.edu
}

\section{Table of Contents}

Nuclear Magnetic Resonance (NMR) Spectroscopy and Mass Spectrometry.............................S2

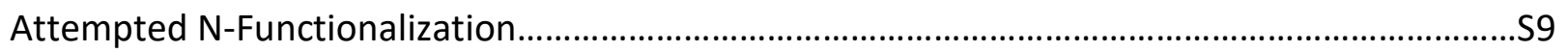

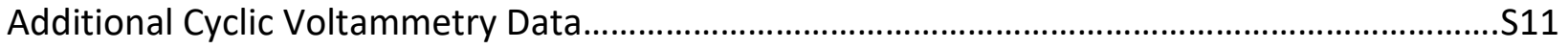

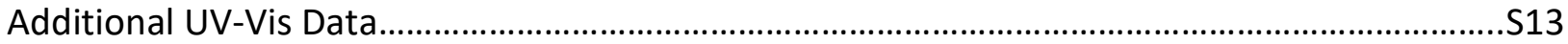

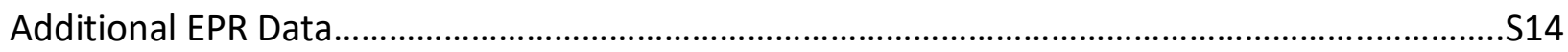

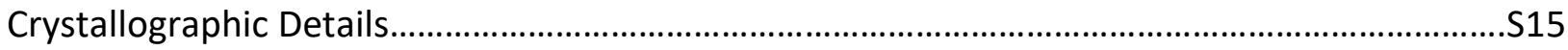

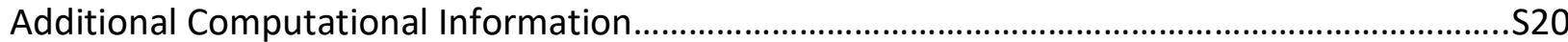

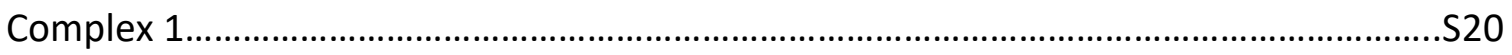

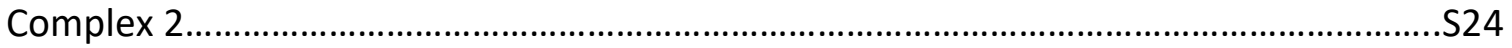

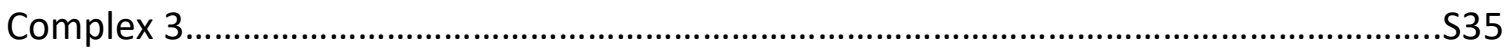

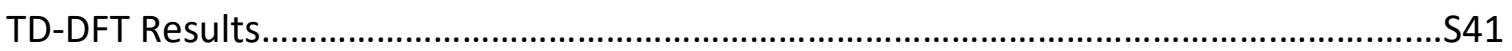

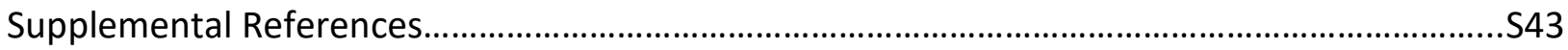




\section{NMR and Mass Spec Characterization for New Compounds}

a

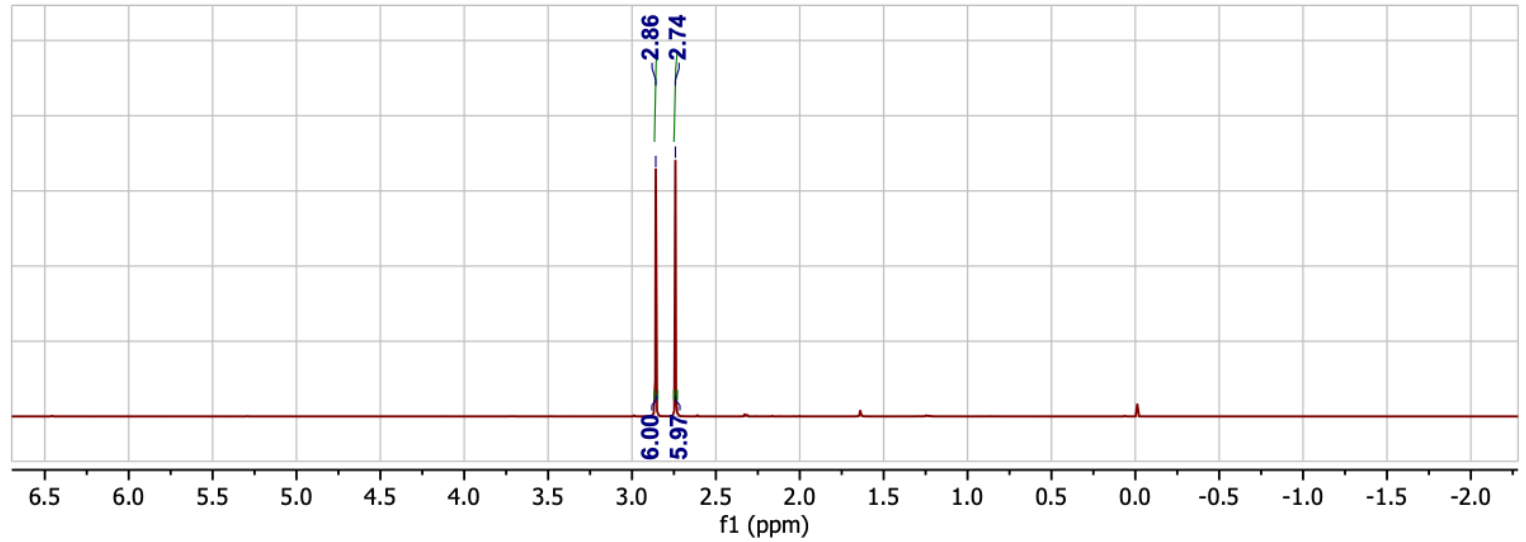

b

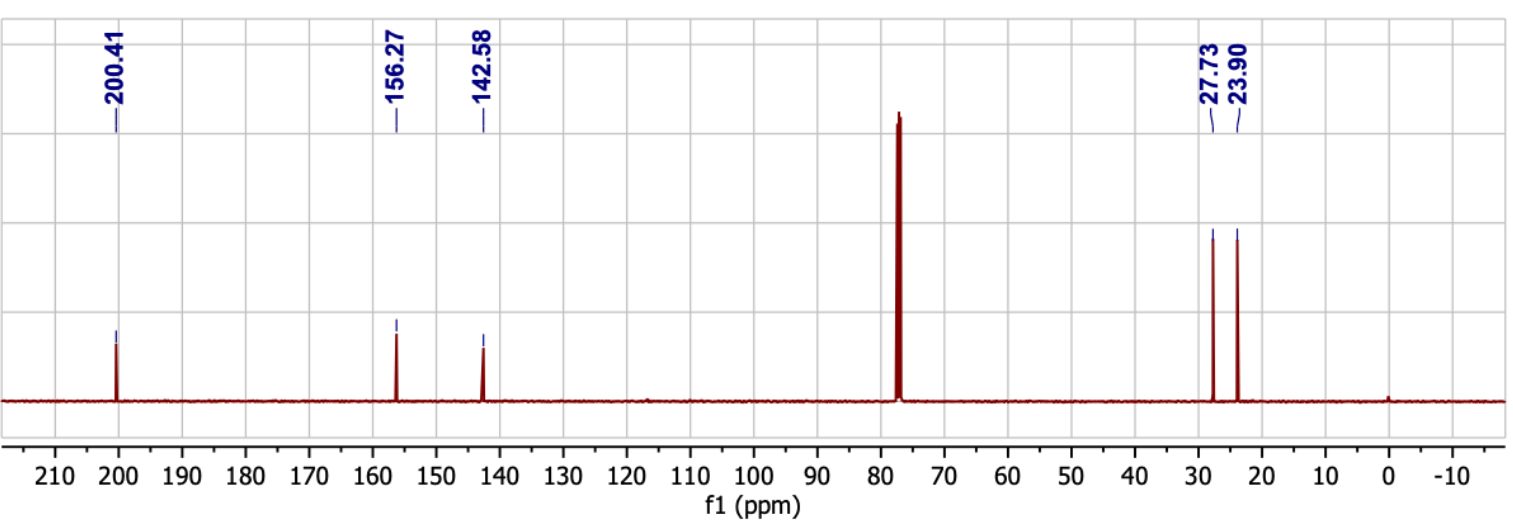

Figure S1. (a) ${ }^{1} \mathrm{H}$ NMR (500 MHz, $298 \mathrm{~K}$ ) and (b) ${ }^{13} \mathrm{C}$ NMR of 3,5-diacetyl-2,6dimethylpyrazine (126 MHz, $298 \mathrm{~K}$ ) in $\mathrm{CDCl}_{3}$ 
a

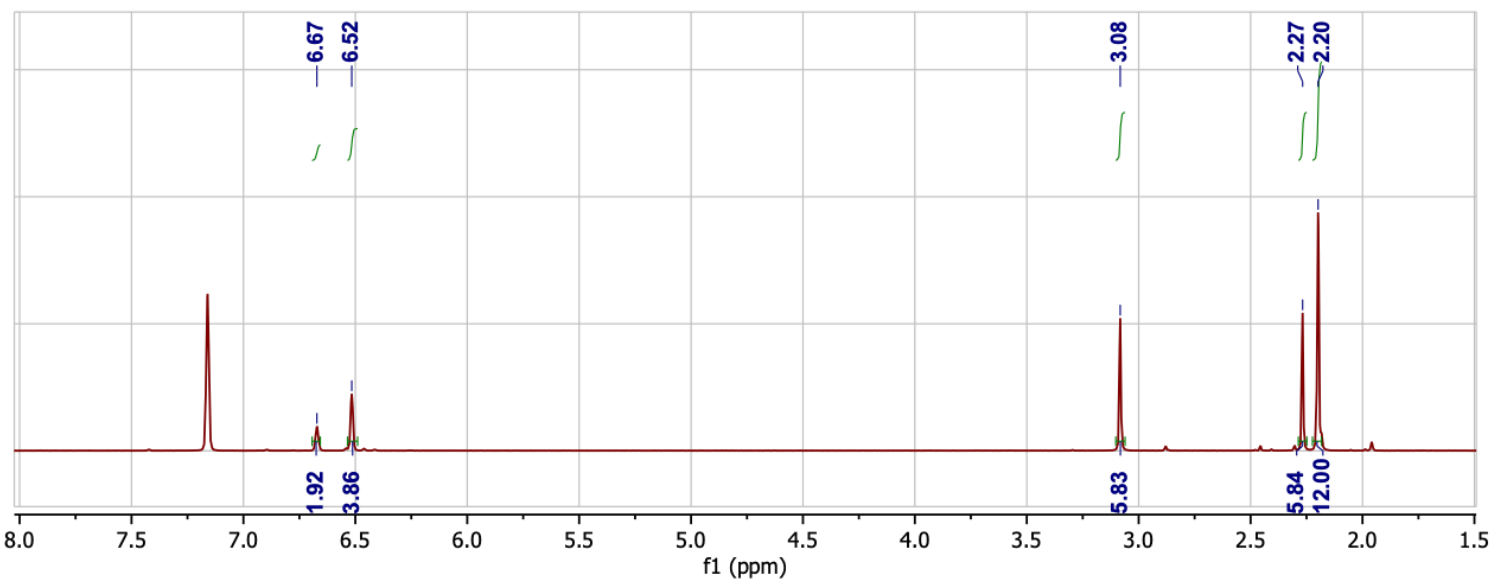

b

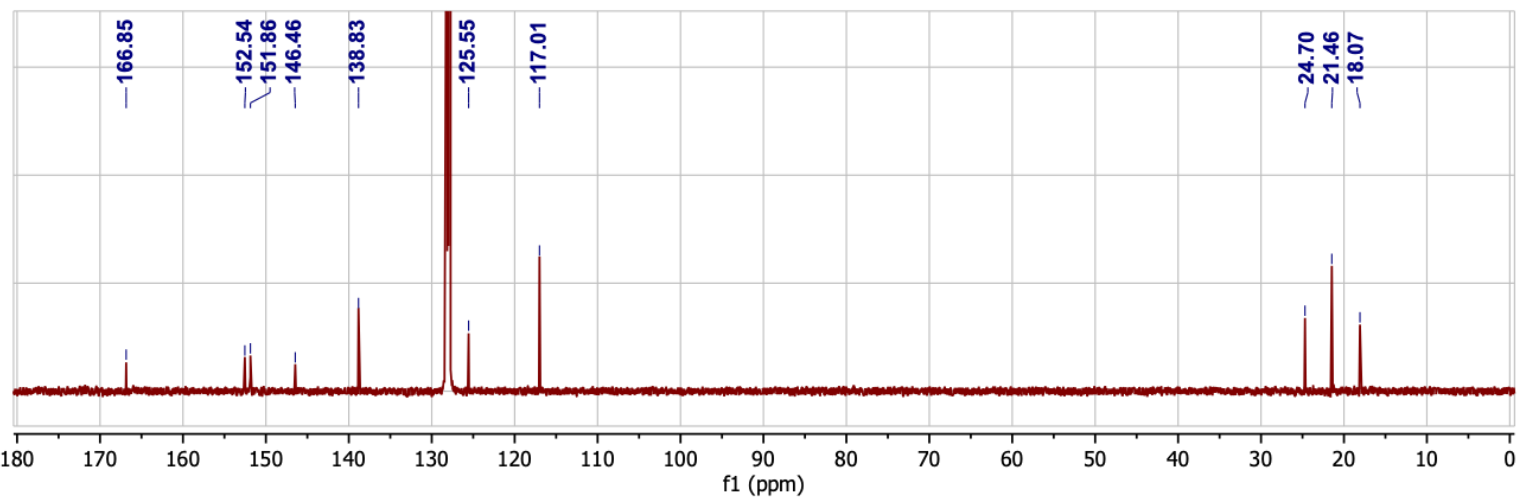

Figure S2. (a) ${ }^{1} \mathrm{H}$ NMR (300 MHz, $\left.298 \mathrm{~K}\right)$ and (b) ${ }^{13} \mathrm{C}$ NMR (126 MHz, $\left.298 \mathrm{~K}\right)$ of 1,1'-(3,5dimethylpyridine-2,6-diyl)bis( $N$-(3,5-dimethylphenyl)ethan-1-imine) (L) in $\mathrm{C}_{6} \mathrm{D}_{6}$.

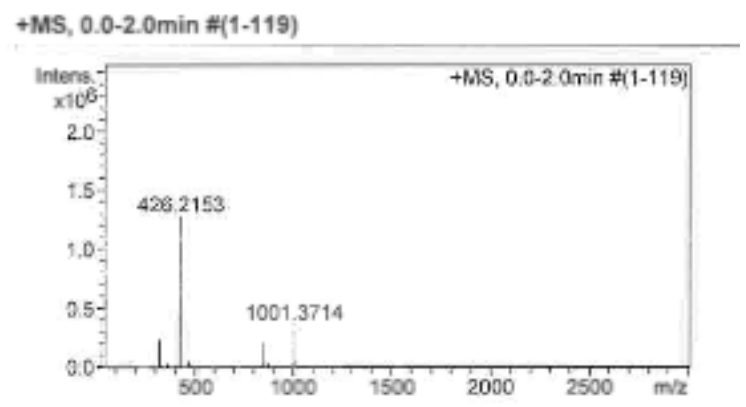

Figure S3. HRMS (ESI) of 1; $426.21 \mathrm{~m} / \mathrm{z}$ peak corresponds to $\left[\mathrm{Fe}(\mathrm{PzDI})_{2}\right]^{2+}$ and $1001.37 \mathrm{~m} / \mathrm{z}$ peak corresponds to $\left[\mathrm{Fe}(\mathrm{PzDI})_{2}(\mathrm{OTf})\right]^{+}$ 
a

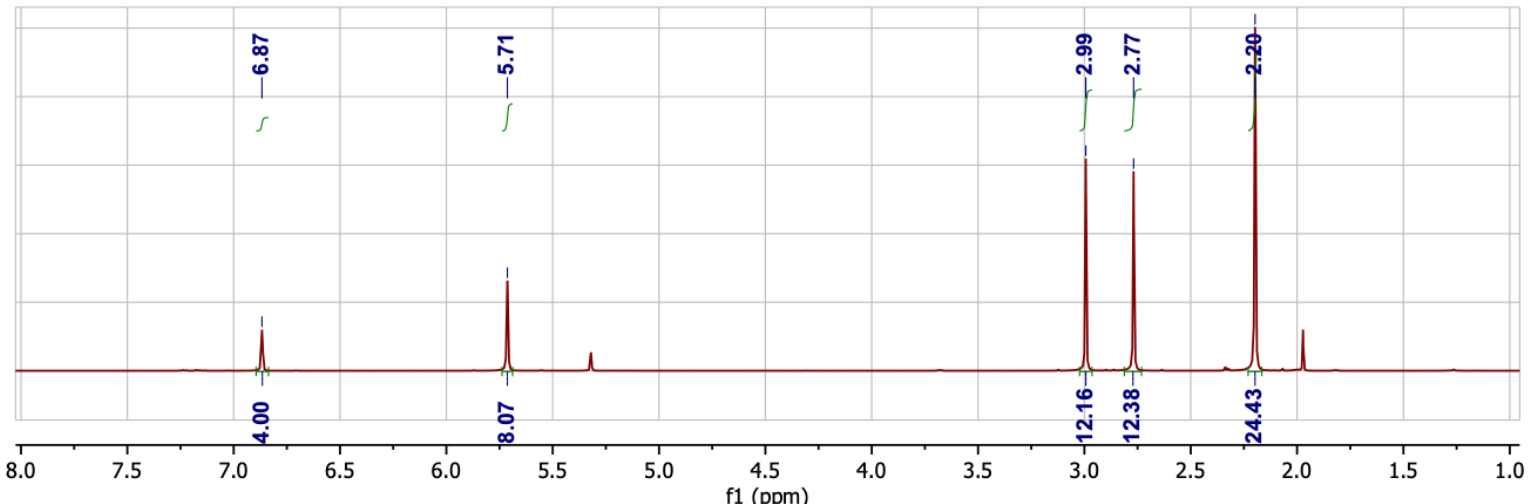

b

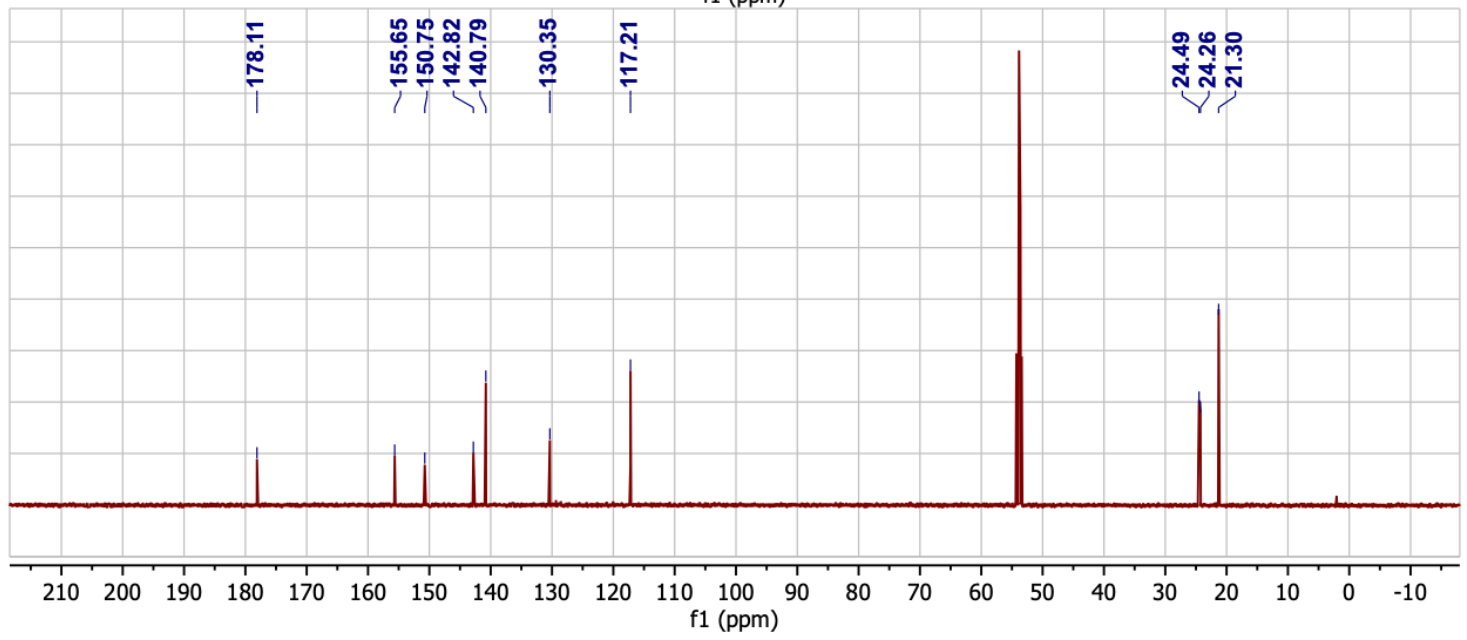

Figure S4. (a) ${ }^{1} \mathrm{H}$ NMR (500 MHz, $\left.298 \mathrm{~K}\right)$ and (b) ${ }^{13} \mathrm{C}$ NMR (126 MHz, $\left.298 \mathrm{~K}\right)$ of 1 in $d_{2}$-methylene chloride 
a
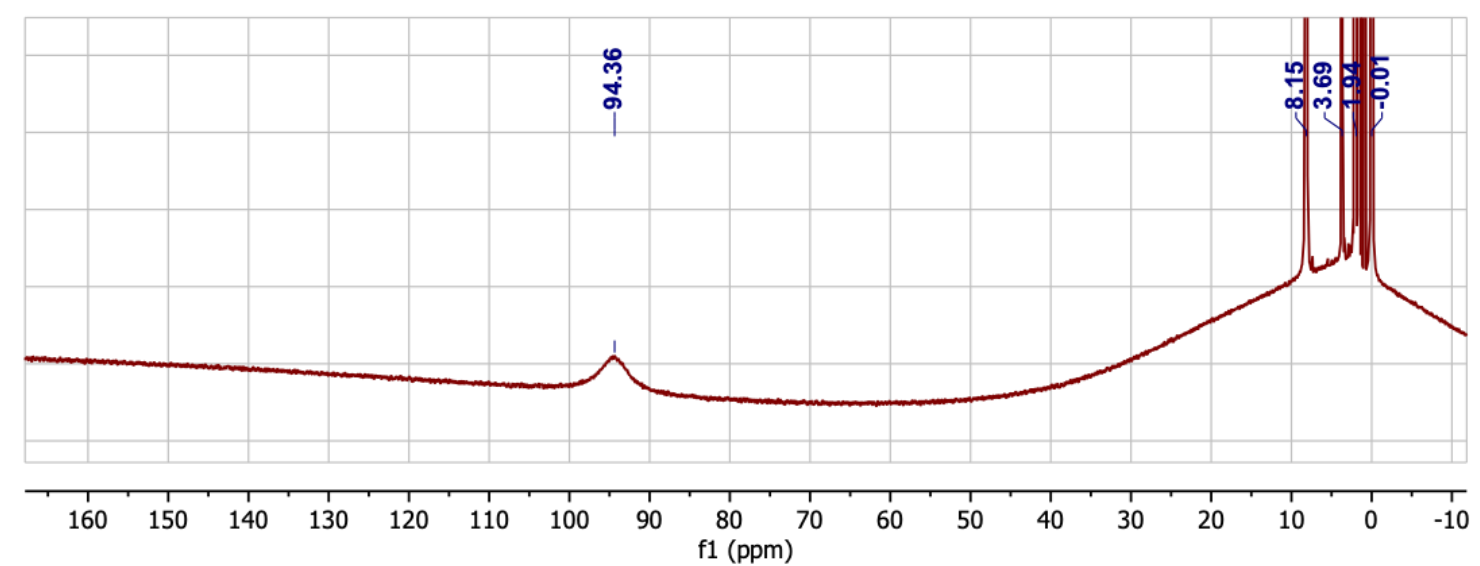

b

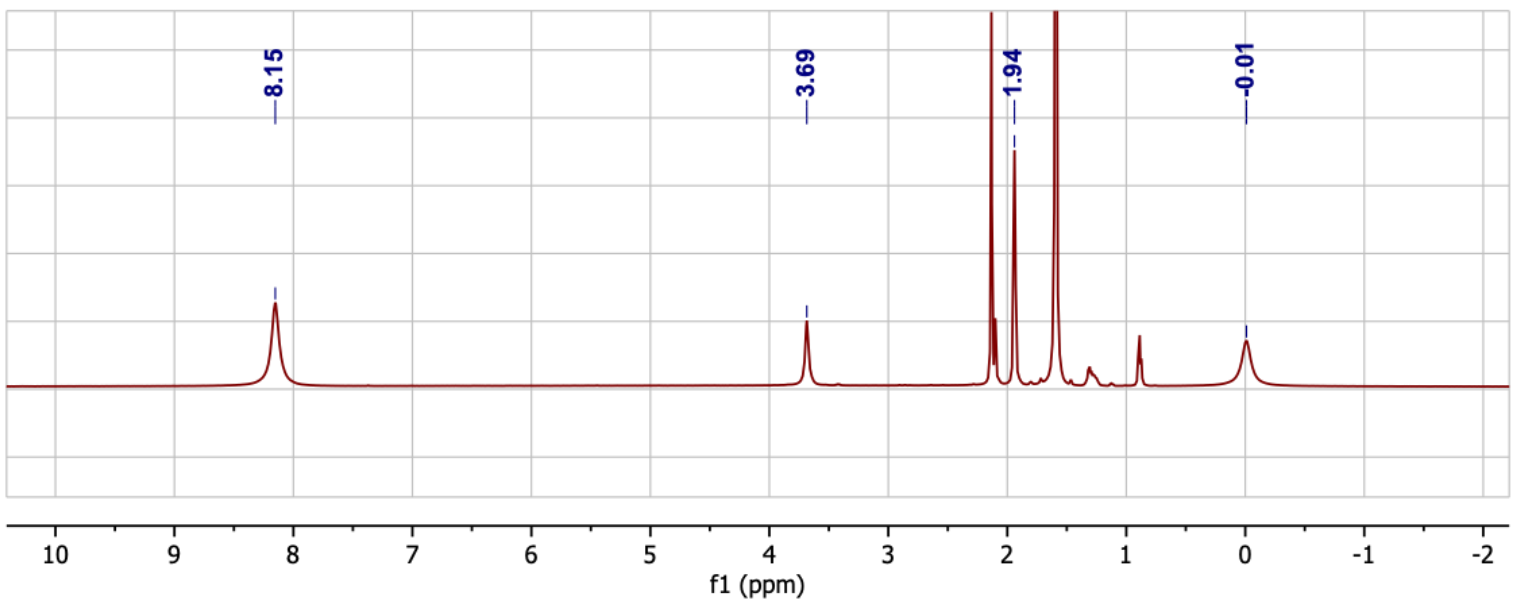

Figure S5. (a) Extended range ${ }^{1} \mathrm{H}$ NMR spectrum ( $300 \mathrm{MHz}, 298 \mathrm{~K}$ ) of $\mathbf{2}$ in $d_{3}$-acetonitrile, showing paramagnetic peak at $94.45 \mathrm{ppm}$. (b) enlarged section of above spectrum near the diamagnetic region. 

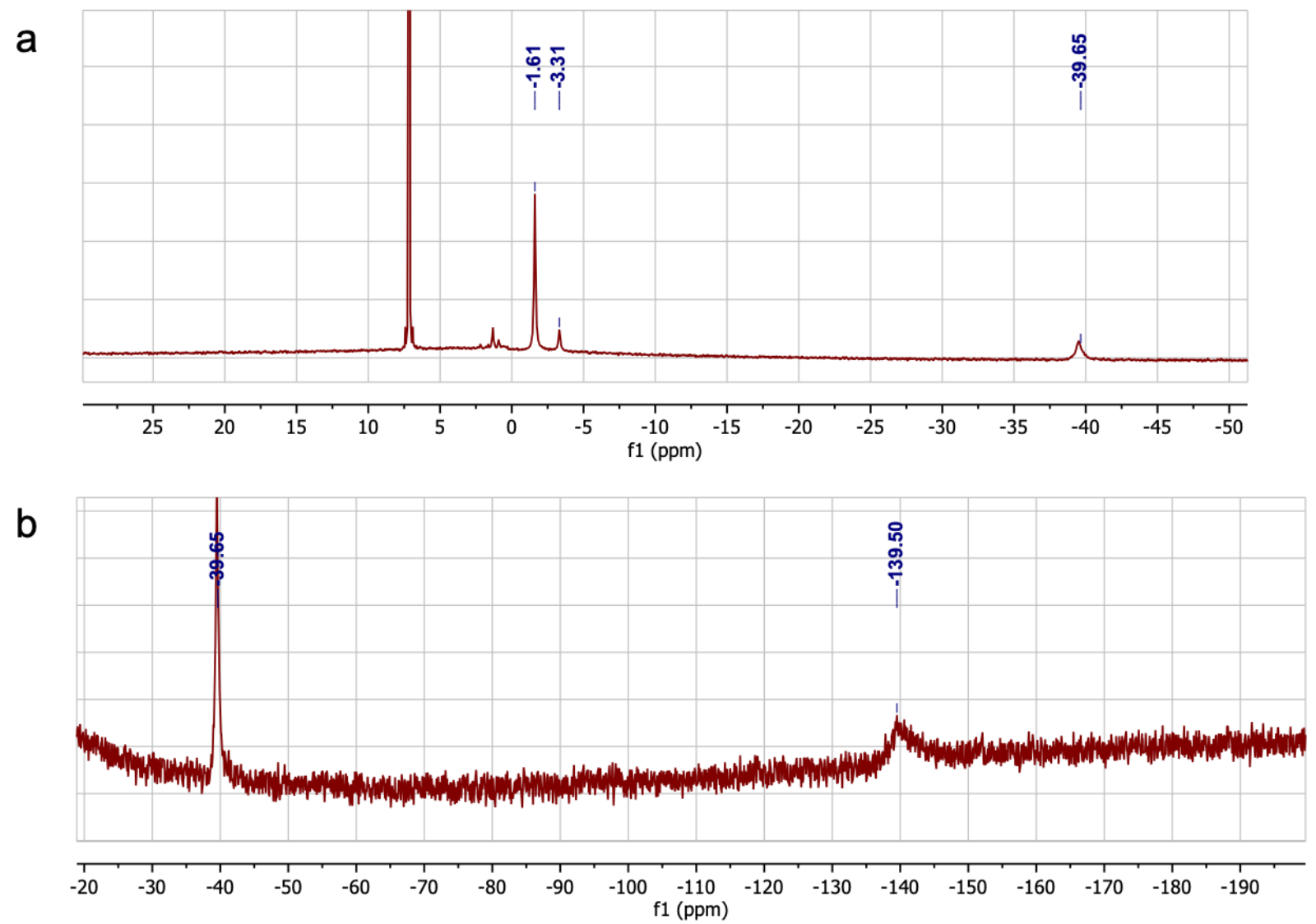

Figure S6. ${ }^{1} \mathrm{H}$ NMR spectrum of 3 in $d_{6}$-benzene ( $300 \mathrm{MHz}, 298 \mathrm{~K}$ ), showing 30 to $-50 \mathrm{ppm}$ (a) and -20 to -200 ppm (b) separately for clarity. 


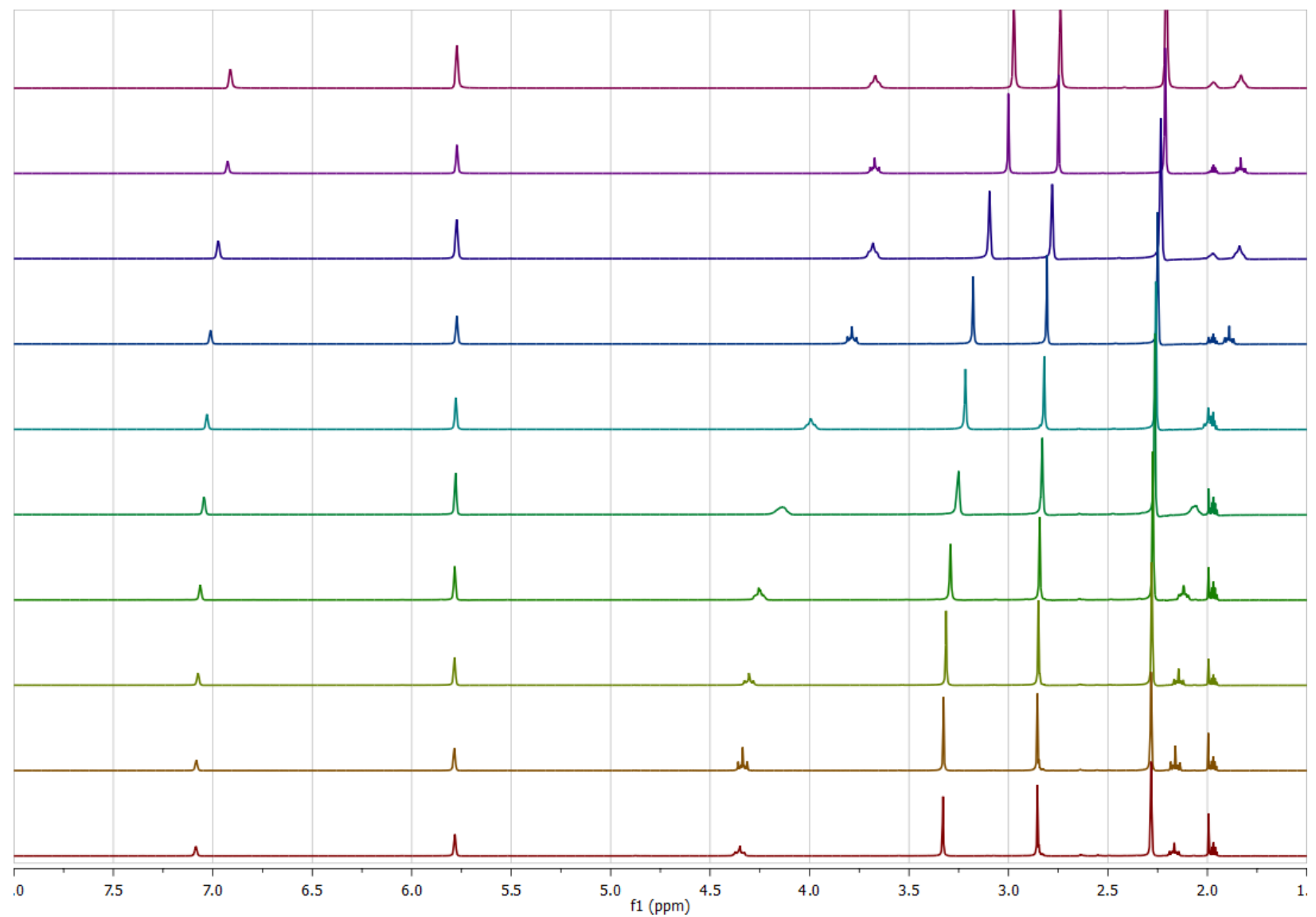

Figure S7. Titration of complex 1 with $0.5 \mathrm{M}$ triflic acid in $d_{3}$-acetonitrile ( $300 \mathrm{MHz}, 298 \mathrm{~K}$ ). Top (purple) shows the complex in the absence of acid, and increasing amounts of acid are added from top to bottom, up to 9 equivalents of HOTf (based on integration of the acidic proton peak at $\sim 15$ ppm, Figure S8). Some residual THF is present.

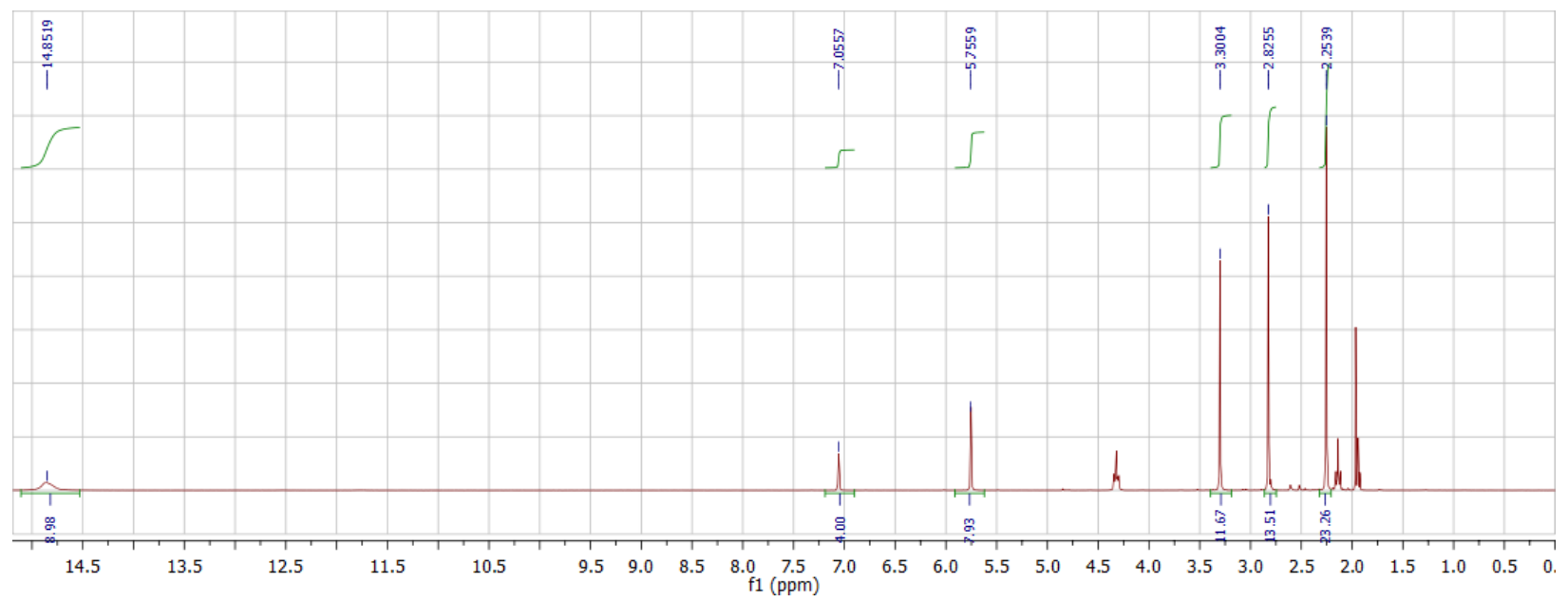

Figure S8. Spectrum of complex 1 after addition of 9 equivalents of triflic acid. 


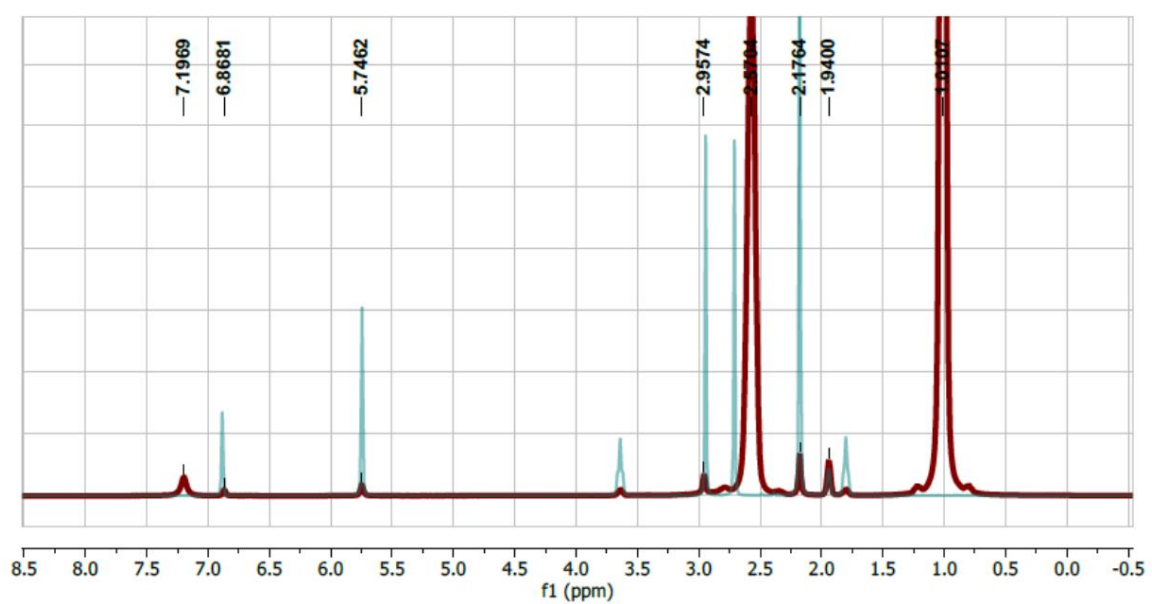

Figure S9. Complex 1 was treated with 2 equivalents of triflic acid and then treated with an excess of triethylamine to confirm the reversibility of the protonation reaction. The spectrum shows the overlay of the result of this reaction (red; peaks at 1.01 and $2.57 \mathrm{ppm}$ are from triethylamine/triethylammonium) with the starting material (complex 1, blue) in acetonitrile. $\left({ }^{1} \mathrm{H} N M R, \mathrm{CD}_{3} \mathrm{CN}, 300 \mathrm{MHz}\right)$.
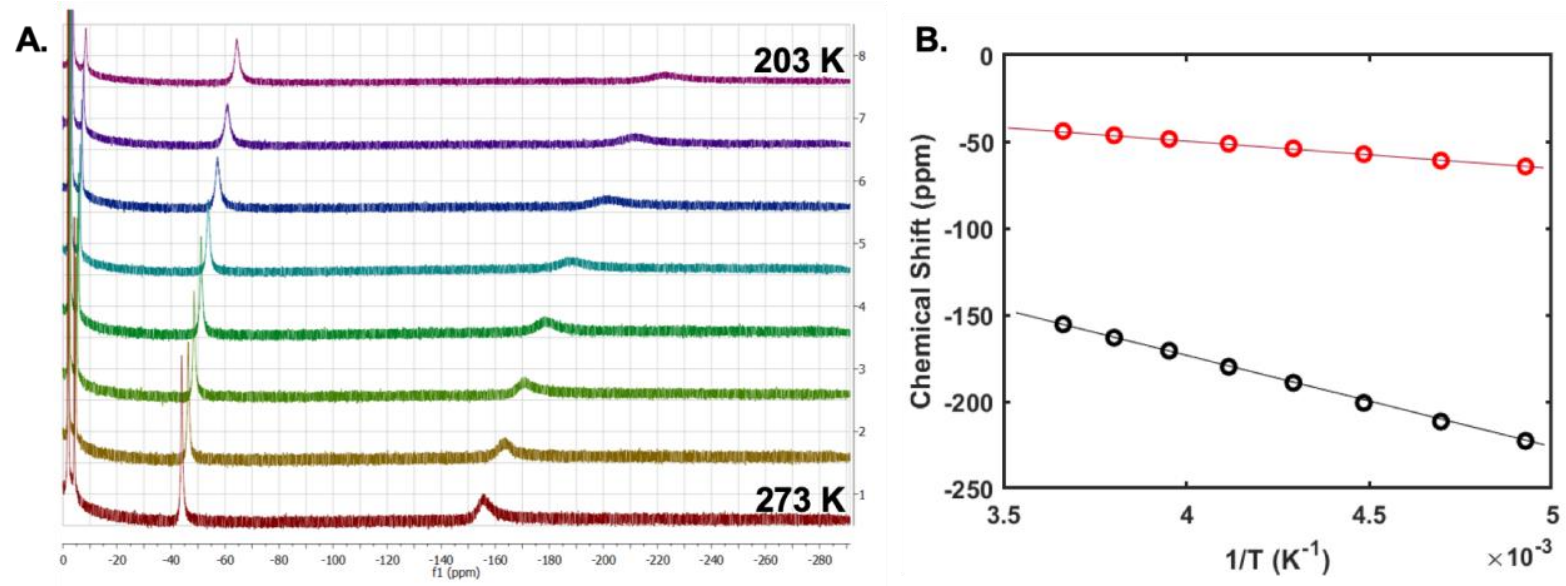

Figure S10. (A) Variable temperature ${ }^{1} \mathrm{H}$ NMR spectra of 3 in $d_{8}$-toluene from $273 \mathrm{~K}$ to $194 \mathrm{~K}$. (B) Chemical shifts in ppm of the two paramagnetically shifted peaks shown in part $A$ vs. change in temperature; linear fit shows that $\mathbf{3}$ displays ideal Curie-Weiss behavior. 


\section{Attempted functionalization of the uncoordinated pyrazine para nitrogen with Lewis} acids and electrophiles

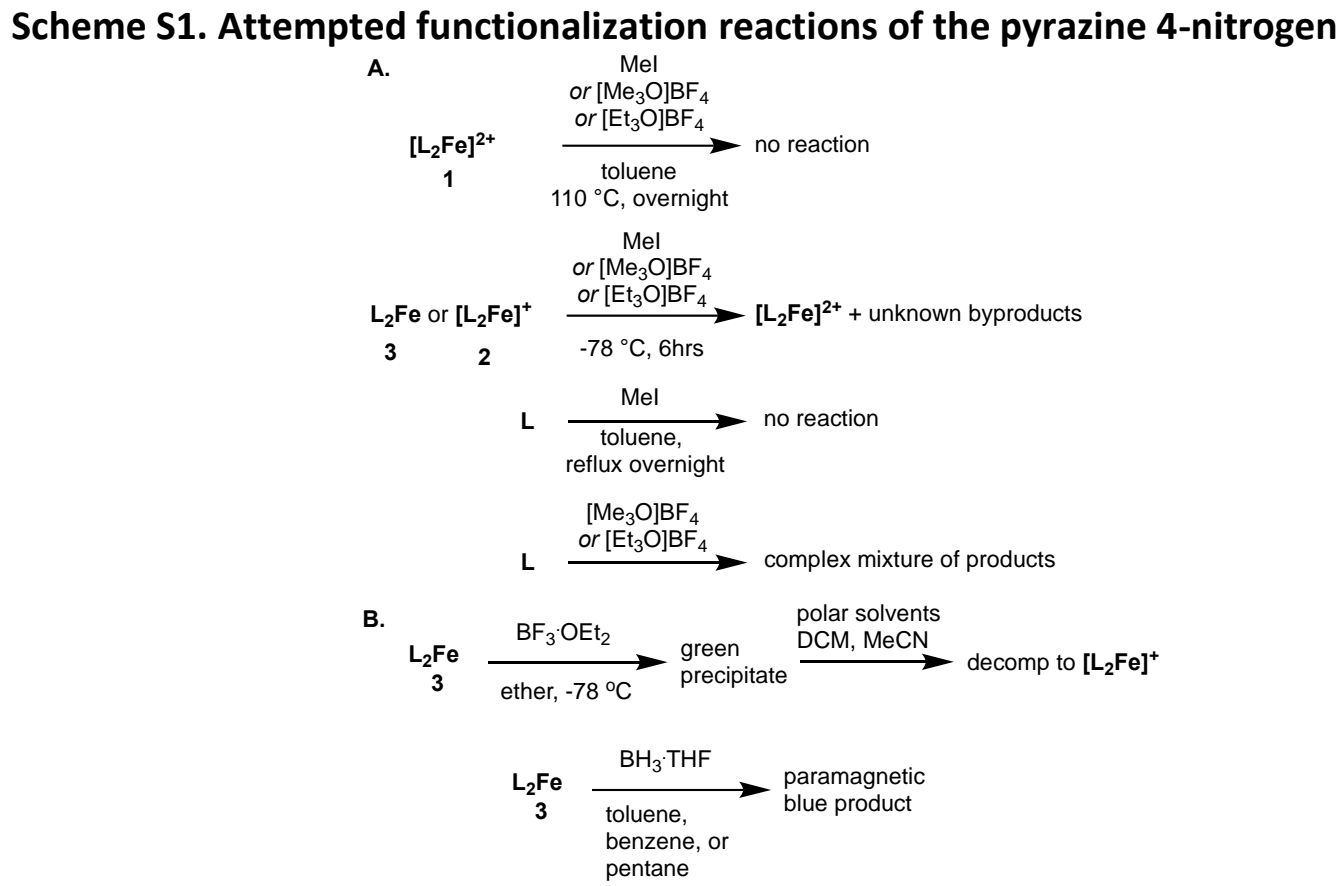

We attempted to functionalize the pyrazine(diimine) ligands through reaction with boronbased Lewis acids. Reaction of the reduced $\mathrm{L}_{2} \mathrm{Fe}$ complex $(3)$ in ether with $\mathrm{BF}_{3} \cdot \mathrm{OEt}_{2}$ at $-78^{\circ} \mathrm{C}$ resulted in the rapid precipitation of a green precipitate which is virtually insoluble in THF and unstable in more polar solvents (DCM, MeCN), decomposing to produce the oxidized complex 1. In contrast, reaction of complex $\mathbf{3}$ in toluene, benzene, or pentane with a solution of $\mathrm{BH}_{3}$ in THF results in formation of a blue product whose NMR spectrum in $\mathrm{C}_{6} \mathrm{D}_{6}$ shows it to be a new paramagnetic product (Figure $\mathrm{S} 11$ ). Unfortunately, crystallographic characterization of both of these products has been elusive; further study of these processes is ongoing.

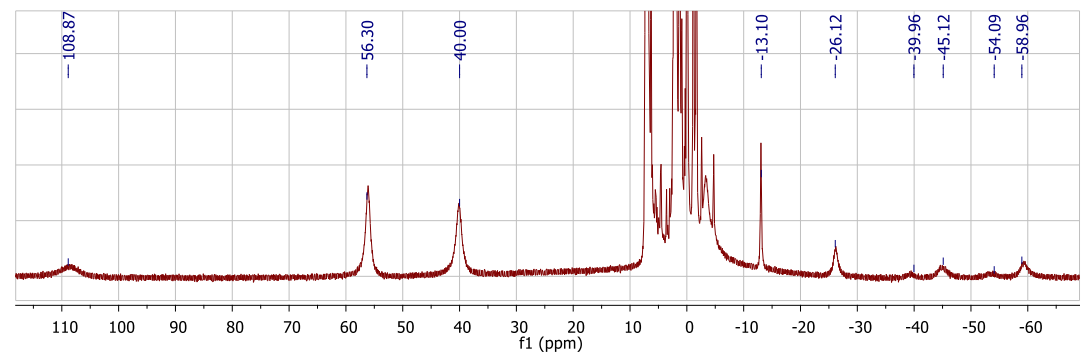

Figure S11. Result of the reaction between complex $\mathbf{3}$ and $\mathrm{BH}_{3} \cdot \mathrm{THF}$. 
a

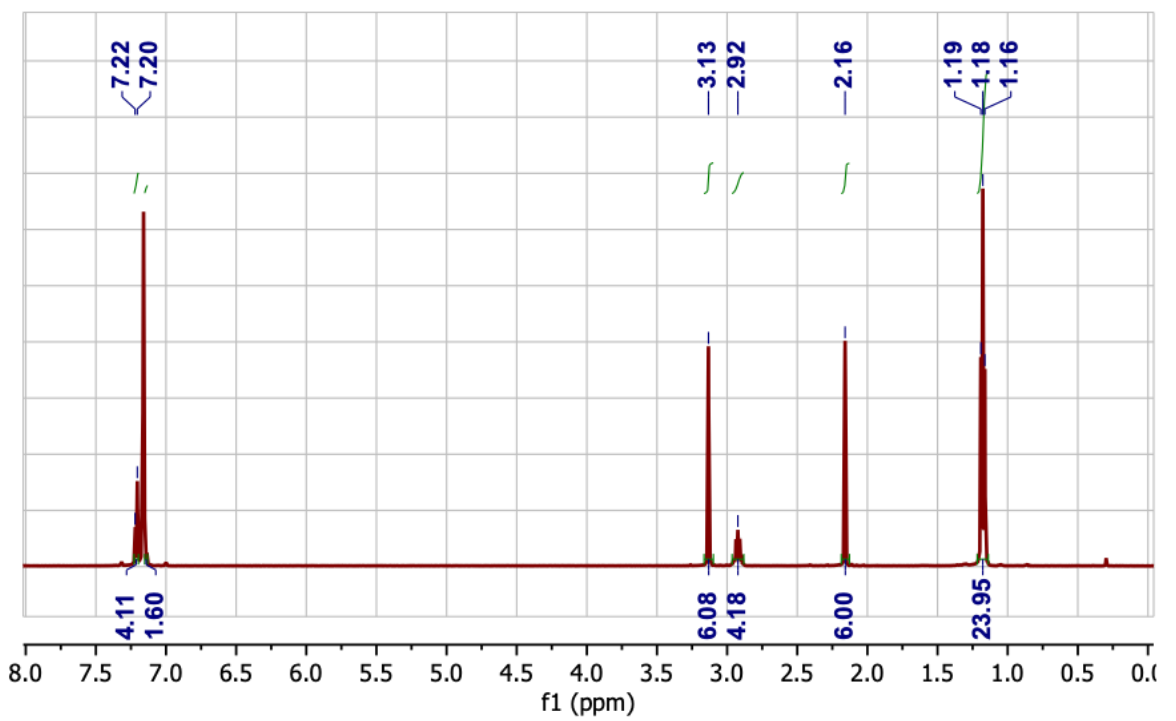

b
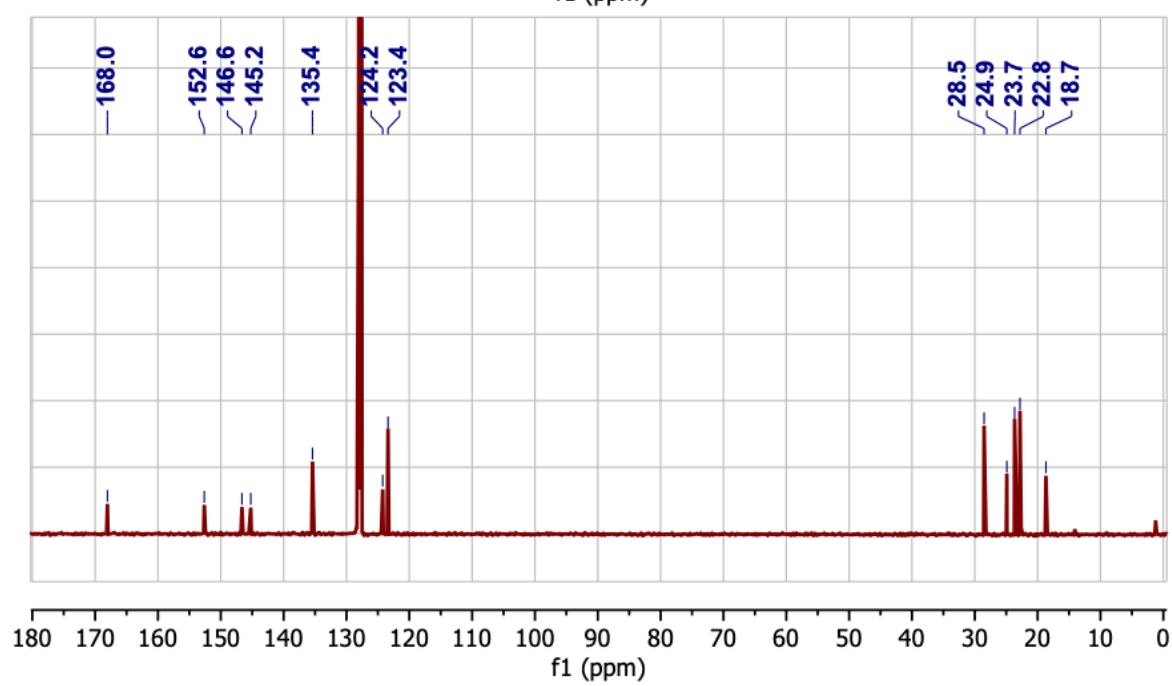

Figure S12. ${ }^{1} \mathrm{H} N M R(a, 300 \mathrm{MHz}, 298 \mathrm{~K})$ and ${ }^{13} \mathrm{C} \mathrm{NMR}(\mathrm{b}, 126 \mathrm{MHz}, 298 \mathrm{~K})$ of ${ }^{2,6-}$ diisopropylphenylpz $\mathrm{DI}$ in $d_{6}$-benzene 


\section{Cyclic Voltammetry}

A.
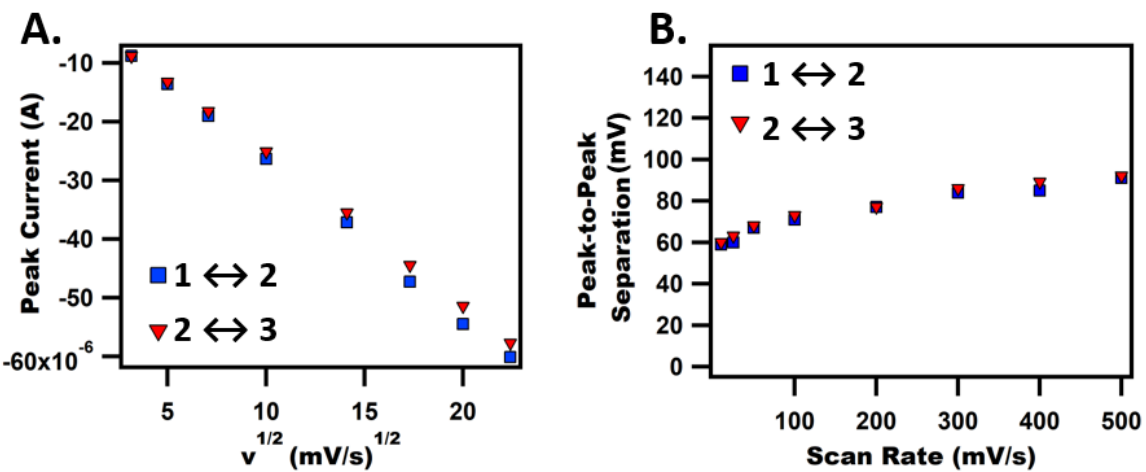

Figure S13. Analysis of variable-scan rate CV scans of complex 1 (see main text Figure 2) confirms that both redox processes (interconversion of $\mathbf{1} \leftrightarrow \mathbf{2}$ and $\mathbf{2} \leftrightarrow \mathbf{3}$ ) correspond to the reduction of freely diffusing species, in accordance with the Randles-Sevcik equation ( $A$, scatter plot of the square root of the scan rate $v s$. the current at the cathodic peaks), and are reversible, albeit with a slight increase in peak-to-peak separation at high scan rates which suggests the presence of some uncompensated solution resistance under the conditions of our experiment (B, scatter plot of the scan rate $v s$. the peak-to-peak separation for both reversible redox couples).

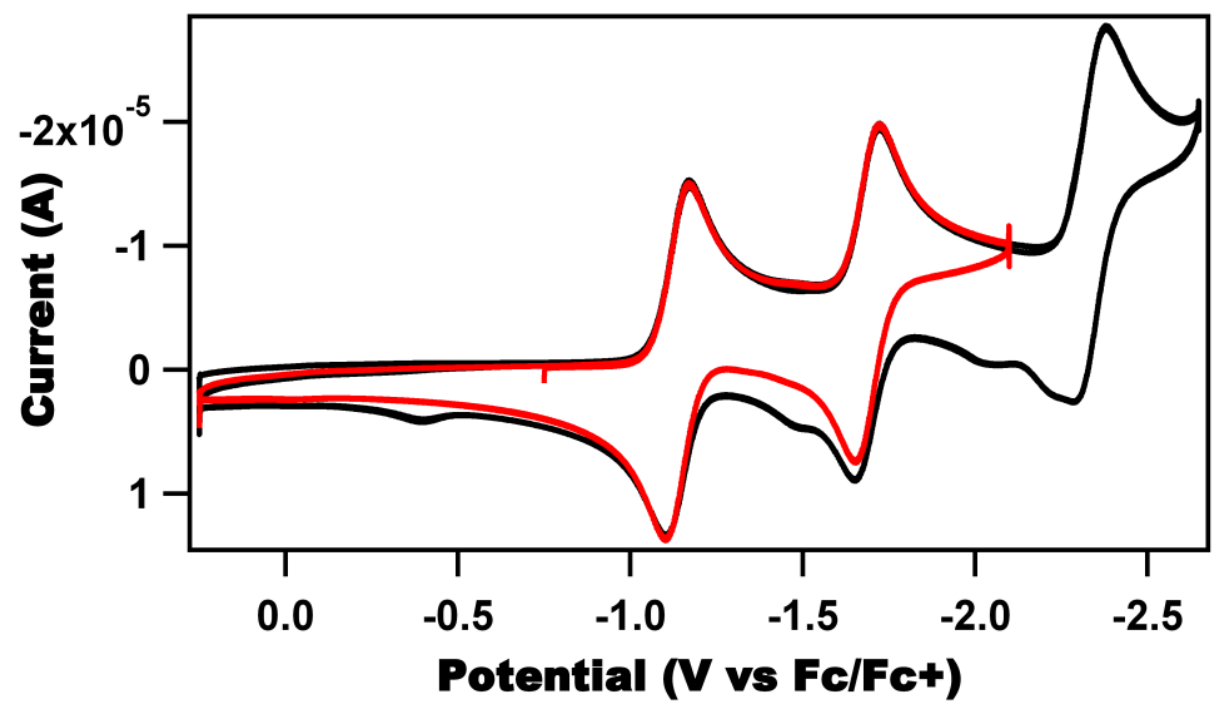

Figure S14. Cyclic voltammogram of complex 1 in $0.1 \mathrm{M} \mathrm{TBAPF}_{6} \mathrm{MeCN}_{\text {solution, showing more }}$ negative partially reversible redox feature (scan rate $100 \mathrm{mV} / \mathrm{s}$ ). Upon scanning past the reduction wave at $-2.5 \mathrm{~V}$, additional oxidative features develop on the return scan. 

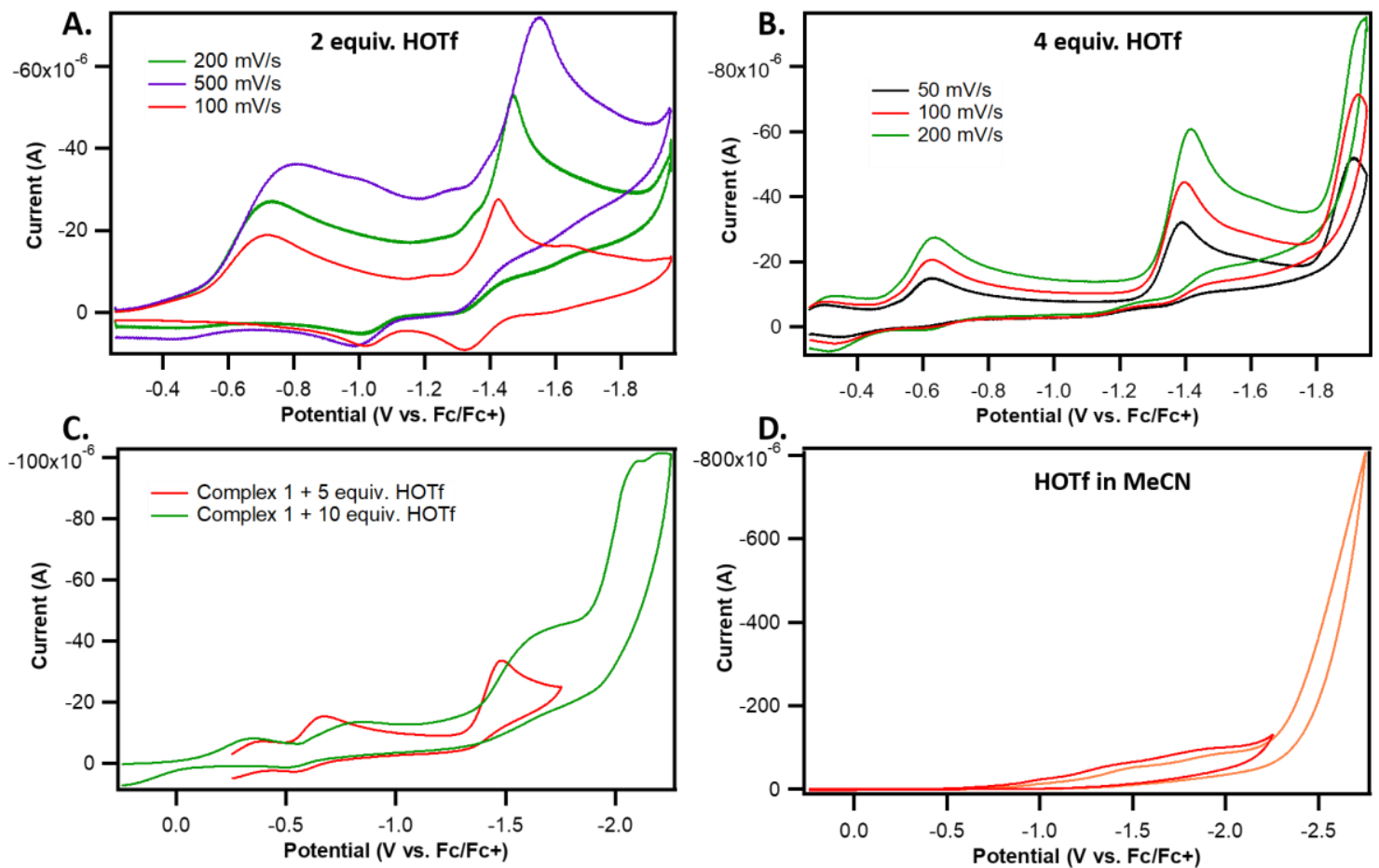

Figure S15. Cyclic voltammograms of complex $1(1 \mathrm{mM})$ in the presence of triflic acid in MeCN $\left(0.1 \mathrm{M} \mathrm{TBAPF}_{6}\right)$. (A) Variable scan rate $\mathrm{CVs}$ of complex 1 in the presence of 2 equiv. of HOTf. (B) Variable scan rate CVs of complex 1 in the presence of 4 equiv. of HOTf. (C) Complex 1 in the presence of 5 or 10 equiv. of HOTf at $100 \mathrm{mV} / \mathrm{s}$. (D) Comparison with blanks of triflic acid (10 $\mathrm{mM}$ ) in $\mathrm{MeCN}$ without complex 1.

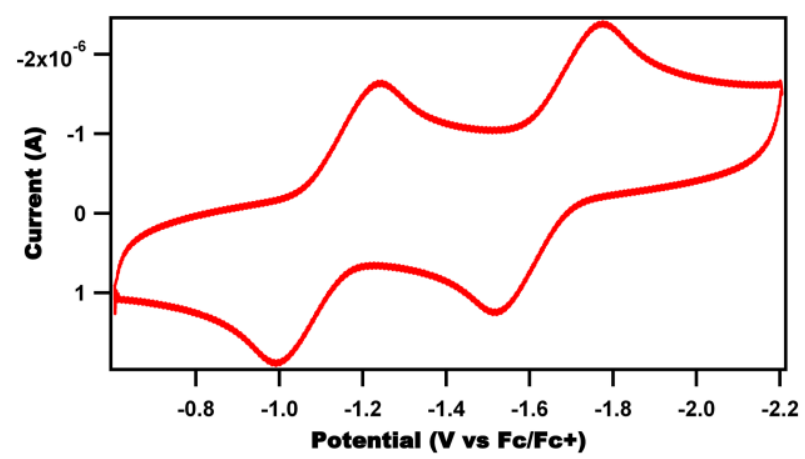

Figure S16. Cyclic voltammogram of complex 1 in THF (0.1 M TBAPF 6 ) shows reduction potentials at $-1.1 \mathrm{~V}$ and $-1.65 \mathrm{~V}$ vs. $\mathrm{Fc} / \mathrm{Fc}^{+}$. 


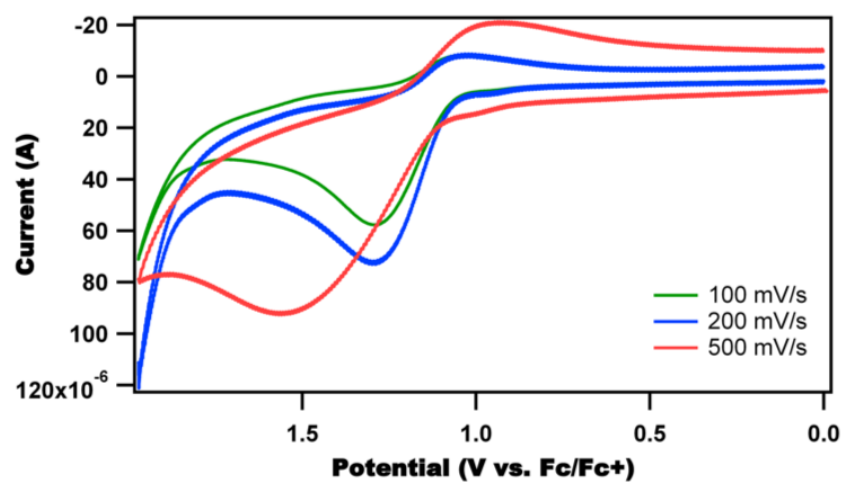

Figure S17. Cyclic voltammogram of complex 1 in acetonitrile (0.1 M TBAPF 6 ) shows an irreversible or partially reversible oxidation peak $\left(E_{p}\right)$ at $\sim 1.2 \mathrm{~V} \mathrm{vs}$. $F c / F^{+}$at $100 \mathrm{mV} / \mathrm{s}$. Initial scan direction was positive.

\section{UV-Vis}

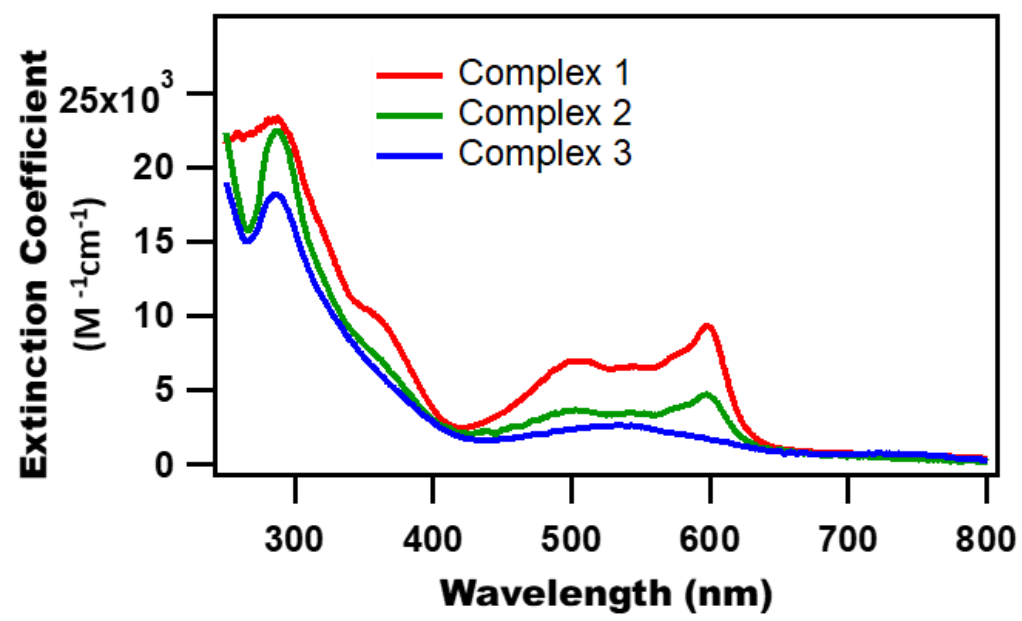

Figure S18. UV-Vis spectra of complexes 1, 2, and 3.

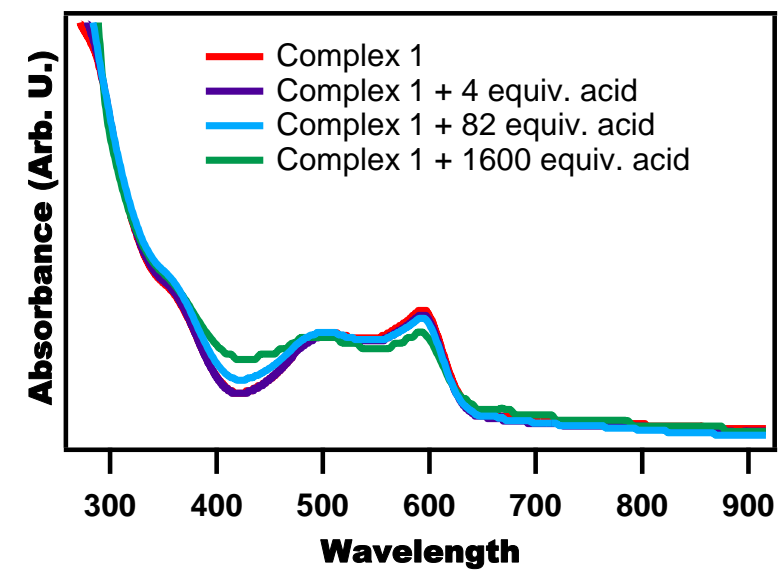

Figure S19. UV-Vis spectra of complex 1 upon treatment with $p$-toluenesulfonic acid in acetonitrile. 


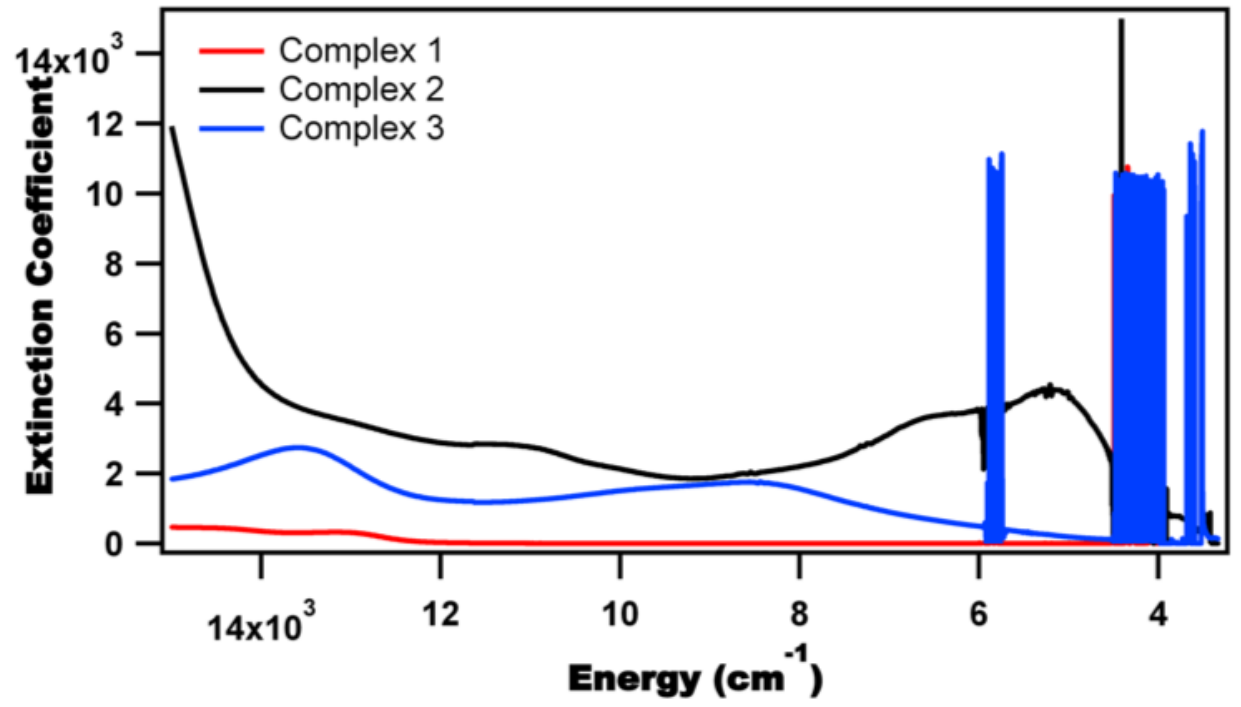

Figure S20. Absorption spectrum of complexes 1-3 in the near-infrared range, in acetonitrile (for 1 and 2) or THF (for 3). Artefacts from solvent absorbance are apparent below $6000 \mathrm{~cm}^{-1}$.

\section{Additional EPR Data}

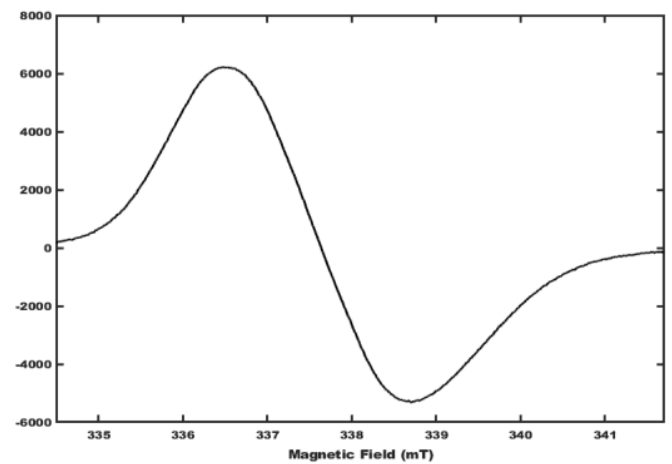

Figure S21. X Band $(9.43 \mathrm{GHz})$ EPR of compound 2 at $286 \mathrm{~K}$.

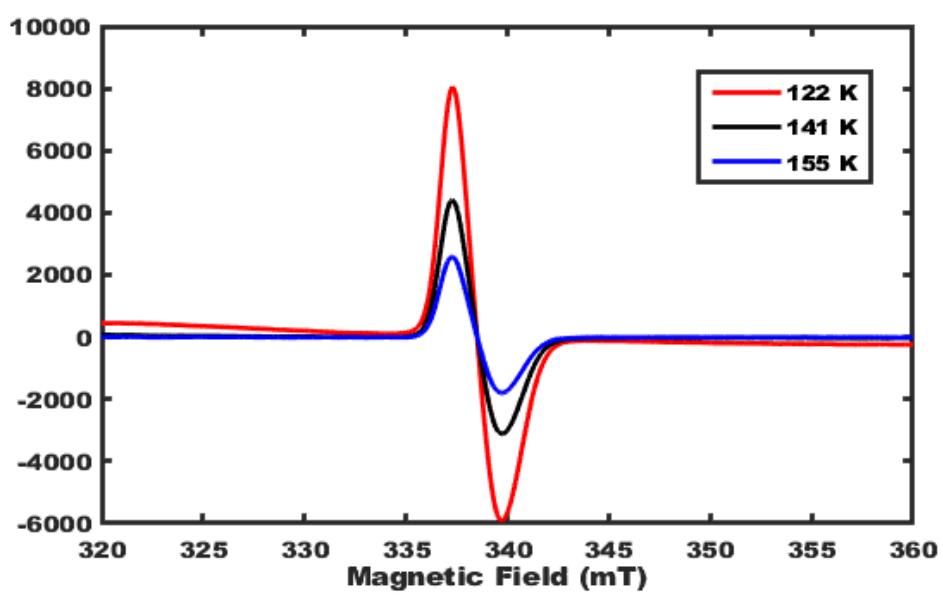

Figure S22. Variable temperature X band EPR $(9.42 \mathrm{GHz})$ of Complex 2. 


\section{Additional Crystallographic Information}

Table S1. Reported Fe-Pyrazine and Fe-Imine Bond Lengths in Pincer Complexes in Highand Low-Spin States

\begin{tabular}{|c|c|c|c|c|}
\hline CCDC ID & Bond Type & Spin State $(S)$ & Length(s) (Å) & Reference \\
\hline FUMLIL & Fe-N(pyrazine) & 2 & 2.042 & 1 \\
\hline DEDNOQ & Fe-N(pyrazine) & 2 & $2.158,2.158$ & 2 \\
\hline DEDNUW & Fe-N(pyrazine) & 2 & $2.146,2.138$ & 2 \\
\hline EMIPIZ01 & Fe-N(pyrazine) & 2 & $2.142,2.156$ & 3 \\
\hline JAFBOI01 & Fe-N(pyrazine) & 2 & $2.124,2.124$ & 4 \\
\hline FUMLOR & Fe-N(pyrazine) & 0 & 1.828 & 1 \\
\hline BAQDON & Fe-N(pyrazine) & 0 & 1.876 & 5 \\
\hline DEDPAE & Fe-N(pyrazine) & 0 & $1.884,1.890$ & 2 \\
\hline EMESUK & Fe-N(pyrazine) & 0 & $1.880,1.865$ & 6 \\
\hline JAFBOI03 & Fe-N(pyrazine) & 0 & $1.898,1.898$ & 4 \\
\hline JAFCID & Fe-N(pyrazine) & 0 & $1.880,1.882$ & 4 \\
\hline SIPFAZ & Fe-N(pyrazine) & 0 & 1.870 & 7 \\
\hline FUMLIL & Fe-N(imine) & 2 & $2.196,2.177$ & 1 \\
\hline BUDPAU & Fe-N(imine) & 2 & $2.251,2.235$ & 8 \\
\hline BOBNUD & Fe-N(imine) & 2 & 2.198 & 9 \\
\hline TULQOH & Fe-N(imine) & 2 & 2.285 & 10 \\
\hline FUMLOR & Fe-N(imine) & 0 & $1.977,1.968$ & 1 \\
\hline BAQDON & Fe-N(imine) & 0 & $1.951,1.984$ & 5 \\
\hline BIPQOJ & Fe-N(imine) & 0 & $1.944,1.948$ & 11 \\
\hline JENDUC & Fe-N(imine) & 0 & $1.927,1.934$ & 12 \\
\hline
\end{tabular}

Table S1 shows representative bond lengths for Fe- $\mathrm{N}_{\text {imine }}$ and $\mathrm{Fe}-\mathrm{N}_{\text {pyrazine }}$ bonds in Fe(II) pincer complexes, tabulated from the Cambridge Crystallographic Data Center (CCDC). Data is separated out into high-spin $(S=2)$ and low-spin $(S=0)$ iron centers, showing that bond lengths can be clearly used to differentiate between those two cases. The data corroborates the conclusion that complex 3 contains a low-spin iron center.

General experimental details. X-ray diffraction (XRD) studies were carried out at the Mississippi State University X-Ray Facility on a Bruker AXS D8 Venture diffractometer using a three-circle goniometer in a kappa geometry with a fixed kappa angle at $54.74^{\circ}$ and equipped with a Photon $100 \mathrm{CMOS}$ active pixel sensor detector. Either monochromatized copper $\operatorname{Ko}(\lambda=1.54178 \AA$ ) or molybdenum $\mathrm{K} \alpha(\lambda=0.71703 \AA)$ radiation was used, as specified below for each structure. Crystals were mounted on a cryoloop using an oil cryoprotectant and the XRD data was collected at low temperature $(T=100 \mathrm{~K})$. All frames were integrated with the aid of the Bruker SAINT software using a narrow-frame algorithm. ${ }^{13}$ Data were corrected for absorption effects using the multi-scan method (SADABS). ${ }^{13}$ The structure was solved using SHELXT, ${ }^{14}$ and was refined against weighted $F^{2}$ values using a full-matrix least-squares procedure with the SHELXTL-2018/3 program suite ${ }^{15}$ as included in the APEX3 Bruker package (2019 version)..$^{13}$ Methyl group hydrogens were 
placed at calculated positions starting from the point of maximum electron density. All other hydrogen atoms were placed at geometrically calculated positions and refined using a riding model. The isotropic displacement parameters of the hydrogen atoms were fixed at 1.2 (1.5 for methyl groups) times the $U_{\text {eq }}$ of the atoms to which they are bonded.

[(PzDI) $\left.{ }_{2} \mathrm{Fe}\right](\mathbf{O T f})_{\mathbf{2}}$ (1). Complex 1 crystallized as purple prisms from the vapor diffusion of ether into an THF solution. A single crystal of approximate dimensions $(0.109 \times 0.234 \times 0.345) \mathrm{mm}^{3}$ was used for the analysis; molybdenum Ka radiation was used. The integration of the data using a monoclinic unit cell yielded a total of 80705 reflections to a maximum $\theta$ angle of $26.37^{\circ}(0.80 \AA$ resolution), of which 12896 were independent (average redundancy 6.2, completeness $=99.9 \%$, $\left.R_{\text {int }}=12.09 \%, R_{\text {sig }}=6.82 \%\right)$ and $8604(66.7 \%)$ were greater than $2 \sigma\left(F^{2}\right)$. The final cell constants of $a=15.22(4) \AA, b=15.68(4) \AA, c=26.93(7) \AA, \beta=100.99(4)^{\circ}$, volume $=6310(30) \AA^{3}$, are based upon the refinement of the XYZ-centroids of 1082 reflections above $20 \sigma$ (I) with $4.898^{\circ}<2 \theta<$ $21.69^{\circ}$. The structure was solved in a monoclinic unit cell with space group $\mathrm{P} 21 / n$ with $\mathrm{Z}=4$ for the formula unit, $\mathrm{C}_{58} \mathrm{H}_{70} \mathrm{~F}_{6} \mathrm{FeN}_{8} \mathrm{O}_{7} \mathrm{~S}_{2}$. Interatomic lengths and angles were restrained and 1,2- and 1,3-rigid bond restraints were applied to all atoms. Two triflate ions were both modeled with static disorder and refined anisotropically with an occupancy ratio of 74:26. One 3,5dimethylphenyl group in the compound was modeled as disordered over two positions in a 74:26 ratio; to aid in modeling the disorder, the atoms of the phenyl groups were constrained to a hexagon, and the atoms of the disordered component were constrained to lie in a flat plane; for some of the atoms that lie at nearly the same position as their disordered partner, the atoms were constrained to have the same anisotropic displacement parameters (EADP). Finally, two disordered diethyl ether molecules were located disordered over two-fold special positions; to aid in modeling these disordered solvent molecules, bond length constraints were applied to the C-C single bonds at a distance of 1.54 Å. Further details are given in Table S1. 

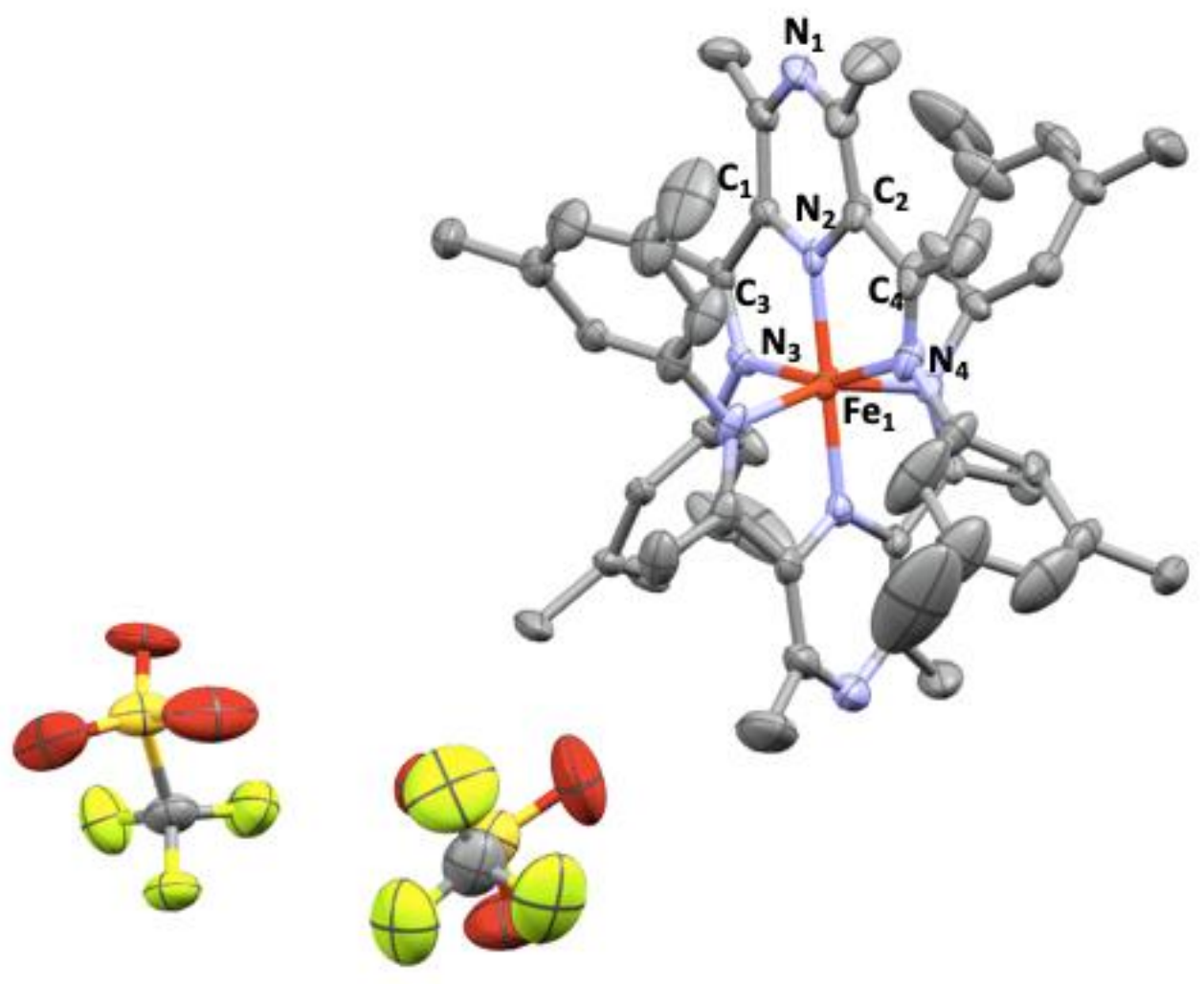

Figure S23. Full asymmetric unit for the X-ray crystal structure of complex 1 . Thermal ellipsoids are shown at $50 \%$ probability; hydrogen atoms, disordered solvent, and minor components of disorder are omitted for clarity.

[(PzDI) ${ }_{2}$ Fe](OTf) (2). Compound $\mathbf{2}$ crystallized as blue-black plates from vapor diffusion of ether into a THF solution. A single crystal with approximate dimensions $(0.098 \times 0.273 \times 0.345) \mathrm{mm}^{3}$ was selected for analysis; copper Ka radiation was used. Data integration with an orthorhombic unit cell yielded a total of 57305 reflections to a maximum $\theta$ angle of $72.44^{\circ}$ ( $0.81 \AA$ A resolution), of which 9630 were independent (average redundancy 5.951 , completeness $=99.9 \%, \mathrm{R}_{\text {int }}=$ $\left.4.41 \%, \mathrm{R}_{\text {sig }}=3.51 \%\right)$ and $9272(96.28 \%)$ were greater than $2 \sigma\left(\mathrm{F}^{2}\right)$. The final cell constants of $a=$ 14.7926(4) $\AA, b=15.4102(4) \AA, c=21.7535(5) \AA$, volume $=4958.9(2) \AA^{3}$, are based upon the refinement of the $X Y Z$-centroids of reflections of 3521 reflections above $20 \sigma(I)$ with $7.027^{\circ}<2 \theta$ $<144.70^{\circ}$. The structure was solved in the chiral space group $\mathrm{P} 2{ }_{1}{ }_{2}{ }_{1}{ }_{1}$ with $\mathrm{Z}=4$ for the formula unit $\mathrm{C}_{53} \mathrm{H}_{60} \mathrm{~F}_{3} \mathrm{FeN}_{8} \mathrm{O}_{3} \mathrm{~S}$. The $\mathrm{CF}_{3}$ group of the triflate anion was modeled anisotropically and with a static disorder with an occupancy ratio of 50:50. Interatomic lengths and angles were restrained and 1,2- and 1,3-rigid bond restraints were applied to aid in modeling the disorder. Further details about the refinement are given in Table S2. 


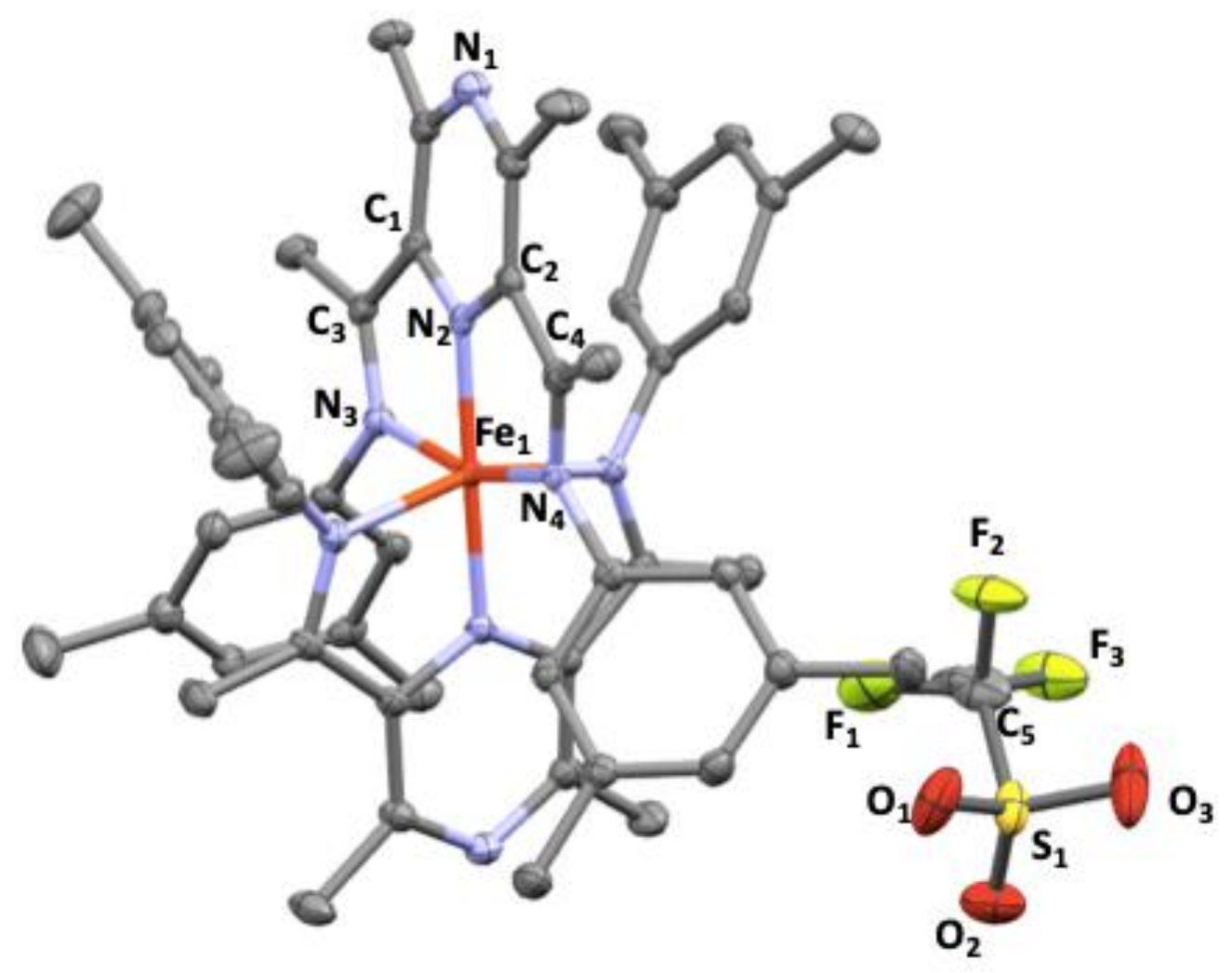

Figure S24. Full asymmetric unit of the X-Ray crystal structure of complex 2. Thermal ellipsoids are shown at 50\% probability; hydrogen atoms and one component of the disordered $\mathrm{CF}_{3}$ group are omitted for clarity.

[(PzDI) ${ }_{2}$ Fe] (3). Dark reddish-brown crystals of $\mathbf{3}$ were grown by slow evaporation of a concentrated pentane solution. A single prism-shaped crystal with approximate dimensions $(0.340 \times 0.0389 \times 0.543) \mathrm{mm}^{3}$ was selected for analysis; molybdenum Ka radiation was used. The integration of the data using a monoclinic unit cell yielded a total of 81248 reflections to a maximum $\theta$ angle of $33.14^{\circ}$ ( $0.65 \AA$ resolution), of which 17025 were independent (average redundancy 4.772 , completeness $\left.=99.4 \%, R_{\text {int }}=4.06 \%, R_{\text {sig }}=3.09 \%\right)$ and $14123(82.95 \%)$ were greater than $2 \sigma \quad\left(F^{2}\right)$. The final cell constants of $a=12.1273(11) \AA, b=21.057(3) \AA, c=17.657(2) \AA, \beta=95.871(4)^{\circ}$, volume $=4485.3(9) \AA^{3}$, are based upon the refinement of the XYZ-centroids of 2212 reflections above $20 \sigma$ (I) with $4.702^{\circ}<$ $2 \theta<74.79^{\circ}$. The structure was solved in the space group $\mathrm{P} 2_{1} / c$ with $\mathrm{Z}=4$ for the formula unit $\mathrm{C}_{52} \mathrm{H}_{60} \mathrm{FeN}_{8}$. Further refinement details are provided in Table S2. An image of the structure is provided in Figure 1 of the main text. 
Table S2. Crystal sample, data, and refinement parameters

\begin{tabular}{|c|c|c|c|}
\hline & Compound 1-Et ${ }_{2} \mathrm{O}$ & Compound 2 & Compound 3 \\
\hline ID code & B_SC_004 & B_SC_010 & B_SC_011 \\
\hline Chemical formula & $\mathrm{C}_{58} \mathrm{H}_{70} \mathrm{~F}_{6} \mathrm{FeN}_{8} \mathrm{O}_{7} \mathrm{~S}_{2}$ & $\mathrm{C}_{53} \mathrm{H}_{60} \mathrm{~F}_{3} \mathrm{FeN}_{8} \mathrm{O}_{3} \mathrm{~S}_{1}$ & $\mathrm{C}_{52} \mathrm{H}_{60} \mathrm{FeN} 8$ \\
\hline Formula weight & $1225.19 \mathrm{~g} / \mathrm{mol}$ & $1002.00 \mathrm{~g} / \mathrm{mol}$ & $852.93 \mathrm{~g} / \mathrm{mol}$ \\
\hline Temperature & $100(2) \mathrm{K}$ & $100(2) \mathrm{K}$ & $100(2) \mathrm{K}$ \\
\hline Wavelength & $0.71073 \AA$ & $1.54178 \AA$ & $0.71073 \AA$ \\
\hline \multirow[t]{2}{*}{ Crystal size } & $(0.109 \times 0.234 \times 0.345)$ & $(0.098 \times 0.273 \times 0.345)$ & $(0.340 \times 0.389 x$ \\
\hline & $\mathrm{mm}^{3}$ & $\mathrm{~mm}^{3}$ & $0.543) \mathrm{mm}^{3}$ \\
\hline Crystal system & Monoclinic & Orthorhombic & Monoclinic \\
\hline Space group & $\mathrm{P} 2{ }_{1} / n$ & $\mathrm{P} 2{ }_{1} 2_{1} 2_{1}$ & $\mathrm{P} 2{ }_{1} / \mathrm{c}$ \\
\hline$a(\AA)$ & $15.22(4)$ & $14.7926(4)$ & $12.1273(11)$ \\
\hline b (Å) & $15.68(4)$ & $15.4102(4)$ & $21.057(3)$ \\
\hline$c(\AA)$ & $26.93(7)$ & $21.7535(5)$ & $17.657(2)$ \\
\hline$\alpha\left({ }^{\circ}\right)$ & 90 & 90 & 90 \\
\hline$\beta\left({ }^{\circ}\right)$ & $100.99(4)$ & 90 & $95.871(4)$ \\
\hline$\gamma\left({ }^{\circ}\right)$ & 90 & 90 & 90 \\
\hline Volume $\left(\AA^{3}\right)$ & $6308(29)$ & $4958.9(2)$ & $4485.3(9)$ \\
\hline $\mathbf{Z}$ & 4 & 4 & 4 \\
\hline Density (calculated) & $1.290 \mathrm{~g} / \mathrm{cm}^{3}$ & $1.342 \mathrm{~g} / \mathrm{cm}^{3}$ & $1.263 \mathrm{~g} / \mathrm{cm}^{3}$ \\
\hline Absorption coefficient & $0.378 \mathrm{~mm}^{-1}$ & $3.353 \mathrm{~mm}^{-1}$ & $0.382 \mathrm{~mm}^{-1}$ \\
\hline$F(000)$ & 2568 & 2108 & 1816 \\
\hline $\begin{array}{l}2 \theta \text { range or data } \\
\text { collection }\end{array}$ & 1.88 to $26.37^{\circ}$ & 3.52 to $72.44^{\circ}$ & 2.17 to $33.14^{\circ}$ \\
\hline Index ranges & $\begin{array}{l}-19<=\mathrm{h}<=19,- \\
19<=\mathrm{k}<=19,-33<=\mid<=33\end{array}$ & $\begin{array}{l}-17<=\mathrm{h}<=18,- \\
19<=\mathrm{k}<=19,-26<=\mathrm{k}<=26\end{array}$ & $\begin{array}{l}-18<=<=18,- \\
32<=k<=32,- \\
25<=\mid<=27\end{array}$ \\
\hline Reflections collected & 80705 & 57305 & 81248 \\
\hline $\begin{array}{l}\text { Independent } \\
\text { reflections }\end{array}$ & $12896[\mathrm{R}$ (int) $=0.1209]$ & $9630[R($ int $)=0.0441]$ & $\begin{array}{l}1702[R \text { (int) }= \\
0.0406]\end{array}$ \\
\hline Absorption correction & Multi-Scan & Multi-Scan & Multi-Scan \\
\hline $\begin{array}{l}\text { Max. and min. } \\
\text { transmission }\end{array}$ & 0.8820 and 0.9610 & 0.7350 and 0.3910 & $\begin{array}{l}0.8810 \text { and } \\
0.8190\end{array}$ \\
\hline $\begin{array}{l}\text { Structure solution } \\
\text { technique }\end{array}$ & Direct methods & Direct methods & Direct methods \\
\hline $\begin{array}{l}\text { Structure solution } \\
\text { program }\end{array}$ & XT, version $2014 / 5$ & $\mathrm{XT}$, version $2014 / 5$ & $\begin{array}{l}X T, \text { version } \\
2014 / 5\end{array}$ \\
\hline Refinement method & $\begin{array}{l}\text { Full-matrix least- } \\
\text { squares on } \mathrm{F}^{2}\end{array}$ & $\begin{array}{l}\text { Full-matrix least- } \\
\text { squares on } \mathrm{F}^{2}\end{array}$ & $\begin{array}{l}\text { Full-matrix least- } \\
\text { squares on } \mathrm{F}^{2}\end{array}$ \\
\hline Refinement program & $\begin{array}{l}\text { SHELXL-2018/3 } \\
\text { (Sheldrick, 2018) }\end{array}$ & $\begin{array}{l}\text { SHELXL-2018/3 } \\
\text { (Sheldrick, 2018) }\end{array}$ & $\begin{array}{l}\text { SHELXL-2018/3 } \\
\text { (Sheldrick, 2018) }\end{array}$ \\
\hline Function minimized & $\sum w\left(F_{o}^{2}-F_{c}^{2}\right)^{2}$ & $\sum w\left(F_{o}^{2}-F_{c}^{2}\right)^{2}$ & $\Sigma w\left(F_{o}^{2}-F_{c}^{2}\right)^{2}$ \\
\hline Data/restraints/param & $12896 / 1863 / 985$ & $9630 / 126 / 675$ & $17025 / 0 / 567$ \\
\hline
\end{tabular}




\begin{tabular}{|c|c|c|c|}
\hline Goodness-of-fit on $F^{2}$ & 1.055 & 1.020 & 1.031 \\
\hline Final $R$ indices & $R 1=0.1059, w R 2=$ & $\mathrm{R} 1=0.0314, w R 2=$ & $\mathrm{R} 1=0.0384$ and \\
\hline$[1>=2 \sigma(I)]$ & 0.2856 & 0.0748 & wR2 = 0.0953 \\
\hline $\begin{array}{l}\text { Final R indices [all } \\
\text { data] }\end{array}$ & $\begin{array}{l}\mathrm{R} 1=0.1494, \mathrm{wR} 2= \\
0.3168\end{array}$ & $\begin{array}{l}\mathrm{R} 1=0.0334, w R 2= \\
0.0764\end{array}$ & $\begin{array}{l}\mathrm{R} 1=0.0517 \text { and } \\
\mathrm{wR} 2=0.1058\end{array}$ \\
\hline Weighting scheme & $\begin{array}{l}\mathrm{w}=1 /\left[\sigma^{2}\left(\mathrm{~F}_{\mathrm{o}}^{2}\right)+(0.1424 \mathrm{P})^{2}+2\right. \\
1.6363 \mathrm{P}] \\
\text { where } \mathrm{P}=\left(\mathrm{F}_{\mathrm{o}}^{2}+2 \mathrm{~F}_{\mathrm{c}}^{2}\right) / 3\end{array}$ & $\begin{array}{l}\mathrm{W}=1 /\left[\sigma^{2}\left(\mathrm{~F}_{\mathrm{o}}^{2}\right)+(0.0305 \mathrm{P})^{2}+2 .\right. \\
7661 \mathrm{P}] \\
\text { where } \mathrm{P}=\left(\mathrm{F}_{\mathrm{o}}^{2}+2 \mathrm{~F}_{\mathrm{c}}^{2}\right) / 3\end{array}$ & $\begin{array}{l}\mathrm{W}=1 /\left[\sigma^{2}\left(\mathrm{~F}_{\mathrm{o}}^{2}\right)+(0.0458\right. \\
\left.\mathrm{P})^{2}+2.6203 \mathrm{P}\right] \\
\text { where } \mathrm{P}=\left(\mathrm{F}_{\mathrm{o}}^{2}+2 \mathrm{~F}_{\mathrm{c}}^{2}\right) / 3\end{array}$ \\
\hline $\begin{array}{l}\text { Largest diff. peak and } \\
\text { hole }\end{array}$ & 1.794 and $-1.079 \mathrm{e}^{-3}$ & 0.680 and $-0.496 \mathrm{e}^{-3}$ & $\begin{array}{l}0.618 \text { and }-0.625 \\
e^{-} \AA^{-3}\end{array}$ \\
\hline $\begin{array}{l}\text { R.M.S. deviation from } \\
\text { mean }\end{array}$ & $0.137 \mathrm{e}^{-3}$ & $0.047 \mathrm{e}^{-3}$ & $0.075 \mathrm{e}^{-3}$ \\
\hline Flack parameter & N/A & $0.0065(15)$ & $\mathrm{N} / \mathrm{A}$ \\
\hline
\end{tabular}

\section{Additional Computational Information}

Complex $\mathbf{1}$ was calculated as a (spin-restricted) singlet, complex $\mathbf{2}$ as a doublet, and complex $\mathbf{3}$ as a triplet. Wavefunctions were checked for internal and external real instabilities, and frequency calculations were carried out on all optimized geometries to ensure only real vibrational modes, indicating true minima. Calculated structures and surfaces were visualized with AGUI from Semichem, Inc.

\section{Supplementary Computational Results for Complex 1}

Table S3. Calculated Coordinates of Complex 1

\begin{tabular}{lrrrr} 
Atom & $\mathrm{X}$ & $\mathrm{Y}$ & $\mathrm{Z}$ \\
\hline Fe1 & 0 & 0.0002 & 0 \\
N2 & 0.336 & -1.4156 & -1.3923 \\
N3 & 1.893 & 0.0009 & -0.0006 \\
N4 & 0.3359 & 1.4162 & 1.392 \\
N5 & -0.3351 & -1.4161 & 1.392 \\
N6 & -1.893 & -0.0003 & 0.0006 \\
N7 & -0.3368 & 1.4163 & -1.3917 \\
N8 & 4.592 & 0.0016 & -0.0012 \\
N9 & -4.592 & -0.0011 & 0.0013 \\
C10 & 1.5907 & -1.6878 & -1.6081 \\
C11 & 2.5362 & -0.8759 & -0.7964 \\
C12 & 3.9548 & -0.898 & -0.7559 \\
C13 & 3.9547 & 0.9008 & 0.7537 \\
C14 & 2.5361 & 0.8781 & 0.7948 \\
C15 & 1.5905 & 1.6894 & 1.607 \\
C16 & -1.5896 & -1.689 & 1.6078 \\
C17 & -2.5356 & -0.8776 & 0.7962 \\
C18 & -3.9542 & -0.9006 & 0.7557
\end{tabular}




\begin{tabular}{|c|c|c|c|}
\hline C19 & -3.9552 & 0.8987 & -0.7534 \\
\hline $\mathrm{C} 20$ & -2.5366 & 0.8767 & -0.7947 \\
\hline $\mathrm{C} 21$ & -1.5915 & 1.6887 & -1.6067 \\
\hline $\mathrm{C} 22$ & -0.7134 & -2.106 & -2.0918 \\
\hline $\mathrm{C} 23$ & -1.139 & -3.3675 & -1.6561 \\
\hline $\mathrm{H} 24$ & -0.6465 & -3.8409 & -0.806 \\
\hline $\mathrm{C} 25$ & -2.1799 & -4.039 & -2.3175 \\
\hline $\mathrm{C} 26$ & -2.786 & -3.4134 & -3.4161 \\
\hline $\mathrm{H} 27$ & -3.5963 & -3.9288 & -3.9401 \\
\hline $\mathrm{C} 28$ & -2.3745 & -2.1523 & -3.8723 \\
\hline $\mathrm{C} 29$ & -1.3357 & -1.4986 & -3.1907 \\
\hline $\mathrm{H} 30$ & -0.9944 & -0.5221 & -3.5361 \\
\hline C31 & 0.7123 & 2.1066 & -2.0919 \\
\hline C32 & 1.3323 & 1.4999 & -3.1925 \\
\hline H33 & 0.9894 & 0.5242 & -3.5387 \\
\hline C34 & 2.3708 & 2.1534 & -3.8748 \\
\hline C35 & 2.7844 & 3.4133 & -3.4175 \\
\hline H36 & 3.5945 & 3.9286 & -3.942 \\
\hline C37 & 2.1807 & 4.0382 & -2.3171 \\
\hline C38 & 1.14 & 3.3669 & -1.655 \\
\hline H39 & 0.6494 & 3.8397 & -0.8035 \\
\hline C40 & 0.7147 & -2.1061 & 2.0913 \\
\hline C41 & 1.1408 & -3.3672 & 1.6554 \\
\hline $\mathrm{H} 42$ & 0.6485 & -3.8409 & 0.8054 \\
\hline $\mathrm{C} 43$ & 2.1822 & -4.0384 & 2.3166 \\
\hline C44 & 2.7881 & -3.4125 & 3.4152 \\
\hline $\mathrm{H} 45$ & 3.5988 & -3.9276 & 3.939 \\
\hline C46 & 2.376 & -2.1517 & 3.8715 \\
\hline C47 & 1.3369 & -1.4984 & 3.1902 \\
\hline $\mathrm{H} 48$ & 0.9951 & -0.5221 & 3.5357 \\
\hline C49 & -0.7136 & 2.1056 & 2.0924 \\
\hline C50 & -1.1423 & 3.3656 & 1.6556 \\
\hline H51 & -0.6523 & 3.8388 & 0.804 \\
\hline C52 & -2.1835 & 4.0361 & 2.3179 \\
\hline C53 & -2.7865 & 3.4107 & 3.4184 \\
\hline H54 & -3.5969 & 3.9253 & 3.9431 \\
\hline C55 & -2.3718 & 2.1511 & 3.8756 \\
\hline C56 & -1.3329 & 1.4984 & 3.1931 \\
\hline H57 & -0.9891 & 0.523 & 3.5392 \\
\hline C58 & -4.8399 & -1.8678 & 1.4914 \\
\hline H59 & -4.5638 & -2.9143 & 1.2989 \\
\hline $\mathrm{H} 60$ & -5.8718 & -1.7053 & 1.158 \\
\hline H61 & -4.8051 & -1.7071 & 2.5803 \\
\hline C62 & -2.0622 & -2.6993 & 2.6095 \\
\hline
\end{tabular}




\begin{tabular}{|c|c|c|c|}
\hline $\mathrm{H} 63$ & -2.5771 & -3.5335 & 2.1092 \\
\hline H64 & -2.7841 & -2.2459 & 3.3036 \\
\hline H65 & -1.2263 & -3.1079 & 3.1872 \\
\hline C66 & 3.0232 & -1.5294 & 5.0841 \\
\hline $\mathrm{H} 67$ & 2.772 & -2.0959 & 5.9962 \\
\hline $\mathrm{H} 68$ & 2.6953 & -0.4911 & 5.2398 \\
\hline $\mathrm{H} 69$ & 4.1215 & -1.5364 & 5.0004 \\
\hline $\mathrm{C} 70$ & 2.6213 & -5.4141 & 1.8797 \\
\hline H71 & 2.1979 & -5.6924 & 0.9036 \\
\hline $\mathrm{H} 72$ & 2.3003 & -6.1767 & 2.6091 \\
\hline $\mathrm{H} 73$ & 3.7183 & -5.4832 & 1.8125 \\
\hline C74 & 4.8407 & 1.8676 & 1.4895 \\
\hline H75 & 4.8054 & 1.7074 & 2.5785 \\
\hline H76 & 4.5655 & 2.9143 & 1.2966 \\
\hline $\mathrm{H} 77$ & 5.8726 & 1.7043 & 1.1565 \\
\hline $\mathrm{C} 78$ & 4.841 & -1.8644 & -1.492 \\
\hline $\mathrm{H} 79$ & 5.873 & -1.7008 & -1.1592 \\
\hline $\mathrm{H} 80$ & 4.8055 & -1.704 & -2.581 \\
\hline H81 & 4.5661 & -2.9111 & -1.2992 \\
\hline C82 & 2.0639 & -2.6977 & -2.6099 \\
\hline $\mathrm{H} 83$ & 2.5797 & -3.5314 & -2.1097 \\
\hline H84 & 2.7851 & -2.2437 & -3.3043 \\
\hline H85 & 1.2282 & -3.1071 & -3.1873 \\
\hline C86 & 2.6214 & 5.4132 & -1.8792 \\
\hline $\mathrm{H} 87$ & 2.2001 & 5.6904 & -0.9018 \\
\hline H88 & 2.2992 & 6.1769 & -2.6069 \\
\hline H89 & 3.7185 & 5.4816 & -1.8142 \\
\hline $\mathrm{C} 90$ & 3.0155 & 1.5322 & -5.0892 \\
\hline H91 & 2.7631 & 2.0999 & -6.0001 \\
\hline H92 & 2.6869 & 0.4943 & -5.2455 \\
\hline H93 & 4.114 & 1.5386 & -5.0072 \\
\hline C94 & -3.0217 & -1.5302 & -5.0849 \\
\hline H95 & -2.6944 & -0.4917 & -5.2403 \\
\hline H96 & -4.12 & -1.5379 & -5.0014 \\
\hline H97 & -2.77 & -2.0964 & -5.997 \\
\hline C98 & -2.6187 & -5.415 & -1.8807 \\
\hline H99 & -2.1935 & -5.6941 & -0.9055 \\
\hline $\mathrm{H} 100$ & -2.2994 & -6.1771 & -2.6112 \\
\hline H101 & -3.7155 & -5.4837 & -1.8114 \\
\hline C102 & -4.8418 & 1.8654 & -1.4888 \\
\hline H103 & -4.8068 & 1.7053 & -2.5778 \\
\hline H104 & -4.5668 & 2.912 & -1.2958 \\
\hline H105 & -5.8736 & 1.7016 & -1.1555 \\
\hline C106 & -2.0652 & 2.6991 & -2.6077 \\
\hline
\end{tabular}




$\begin{array}{lrrr}\text { H107 } & -2.581 & 3.5324 & -2.1069 \\ \text { H108 } & -2.7865 & 2.2454 & -3.3022 \\ \text { H109 } & -1.2297 & 3.109 & -3.1852 \\ \mathrm{C} 110 & -3.0158 & 1.5293 & 5.0901 \\ \mathrm{H} 111 & -2.6862 & 0.4917 & 5.2464 \\ \mathrm{H} 112 & -4.1142 & 1.5348 & 5.0084 \\ \mathrm{H} 113 & -2.7637 & 2.0973 & 6.001 \\ \mathrm{C} 114 & -2.6255 & 5.4106 & 1.8801 \\ \mathrm{H} 115 & -2.2029 & 5.689 & 0.9036 \\ \mathrm{H} 116 & -2.3058 & 6.1744 & 2.6088 \\ \mathrm{H} 117 & -3.7226 & 5.4774 & 1.8132 \\ \mathrm{C} 118 & 2.0635 & 2.6999 & 2.6081 \\ \mathrm{H} 119 & 2.5792 & 3.5334 & 2.1074 \\ \mathrm{H} 120 & 2.7849 & 2.2466 & 3.3028 \\ \mathrm{H} 121 & 1.2278 & 3.1096 & 3.1853\end{array}$

Table S4. Selected Kohn-Sham Orbital Occupancies and Energies for Complex 1

\begin{tabular}{|c|c|c|c|}
\hline $\mathrm{MO}$ & Occupancy & $\begin{array}{l}\text { Energy } \\
\text { (Hartrees) }\end{array}$ & $\begin{array}{l}\text { Energy } \\
(\mathrm{eV})\end{array}$ \\
\hline 234 & 0 & -0.20204 & -5.49782 \\
\hline 233 & 0 & -0.2057 & -5.59736 \\
\hline 232 & 0 & -0.20723 & -5.63907 \\
\hline 231 & 0 & -0.21598 & -5.87724 \\
\hline 230 & 0 & -0.2676 & -7.28178 \\
\hline 229 & 0 & -0.27004 & -7.34821 \\
\hline 228 & 0 & -0.28246 & -7.68625 \\
\hline 227 & 0 & -0.28344 & -7.71286 \\
\hline 226 & 2 & -0.40715 & -11.079 \\
\hline 225 & 2 & -0.40736 & -11.0849 \\
\hline 224 & 2 & -0.40892 & -11.1272 \\
\hline 223 & 2 & -0.41688 & -11.3439 \\
\hline 222 & 2 & -0.41694 & -11.3455 \\
\hline 221 & 2 & -0.41798 & -11.3739 \\
\hline 220 & 2 & -0.41826 & -11.3816 \\
\hline 219 & 2 & -0.41829 & -11.3824 \\
\hline 218 & 2 & -0.41909 & -11.404 \\
\hline 217 & 2 & -0.42602 & -11.5925 \\
\hline 216 & 2 & -0.42631 & -11.6006 \\
\hline
\end{tabular}


Supplementary Computational Results for Complex 2

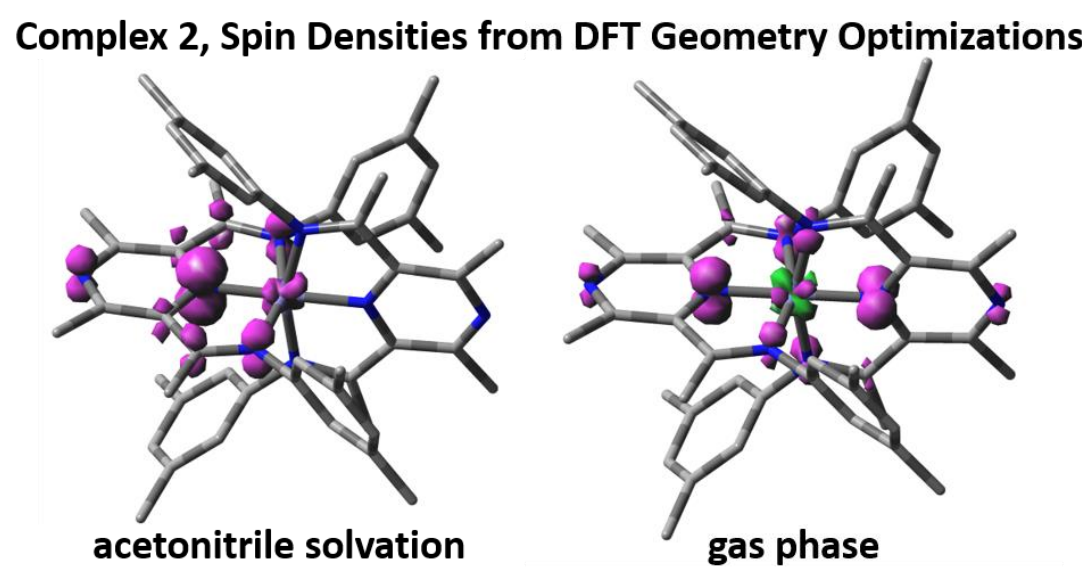

Figure S25. Comparison of the spin densities calculated for complex $\mathbf{2}$ from the DFT-optimized structures using acetonitrile solvation (left) compared to the results without solvation (right). Both are shown at a spin density of $0.008 \mathrm{e} / \AA^{3}$.

Table S5. Calculated Bond Lengths for Complex 2 using C-PCM Solvation with Acetonitrile

\begin{tabular}{|c|c|c|}
\hline Bond & $\mathbf{L}$ & $\mathrm{L}^{\prime}$ \\
\hline $\mathrm{Fe}-\mathrm{N}^{\mathrm{pz}}{ }_{1}$ & 1.900 & 1.875 \\
\hline $\mathrm{Fe}-\mathrm{Nim}_{2}$ & 2.014 & 2.028 \\
\hline $\mathrm{Fe}-\mathrm{Nim}_{3}$ & 2.012 & 2.015 \\
\hline $\mathrm{N}^{\mathrm{im}_{2}-\mathrm{C}^{\mathrm{im}}}{ }_{1}$ & 1.325 & 1.303 \\
\hline $\mathrm{N}^{\mathrm{im}_{3}-\mathrm{C}^{\mathrm{im}}}{ }_{2}$ & 1.327 & 1.303 \\
\hline $\mathrm{C}^{\mathrm{im}_{1}-\mathrm{C}^{\mathrm{pz}} \mathrm{z}_{3}}$ & 1.455 & 1.483 \\
\hline $\mathrm{C}^{\mathrm{im}_{2}-\mathrm{C}^{\mathrm{pz}} 4}$ & 1.452 & 1.484 \\
\hline $\mathrm{Npz}_{1}-\mathrm{C}^{\mathrm{pz}} \mathrm{z}_{3}$ & 1.373 & 1.354 \\
\hline $\mathrm{N}^{\mathrm{pz}} \mathrm{z}_{1}-\mathrm{C}^{\mathrm{pz}}{ }_{4}$ & 1.375 & 1.353 \\
\hline
\end{tabular}

Table S6. Optimized Coordinates for Complex 2 with THF Solvation

\begin{tabular}{l|r|r|r} 
Atom & $\mathrm{X}$ & $\mathrm{Y}$ & $\mathrm{Z}$ \\
\hline Fe1 & 3.9035 & 8.1074 & 5.928 \\
N2 & 5.1568 & 9.5286 & 6.107 \\
N3 & 2.9043 & 9.6016 & 4.99 \\
N4 & 2.7721 & 8.3875 & 7.6067 \\
N5 & 2.7064 & 6.6707 & 5.7513
\end{tabular}




\begin{tabular}{|c|c|c|c|}
\hline N6 & 4.5697 & 7.3306 & 4.2023 \\
\hline N7 & 5.3733 & 7.1827 & 6.9519 \\
\hline N8 & 6.9542 & 11.5651 & 6.3675 \\
\hline N9 & 0.9889 & 4.5866 & 5.4864 \\
\hline C10 & 4.8342 & 10.7664 & 5.6063 \\
\hline C11 & 5.765 & 11.8104 & 5.7884 \\
\hline C12 & 7.2887 & 10.3347 & 6.8044 \\
\hline C13 & 6.3659 & 9.2771 & 6.707 \\
\hline C14 & 6.4589 & 7.9066 & 7.1819 \\
\hline C15 & 3.9606 & 6.2485 & 3.8089 \\
\hline C16 & 2.8603 & 5.8245 & 4.7058 \\
\hline C17 & 1.9829 & 4.719 & 4.601 \\
\hline C18 & 0.8199 & 5.4383 & 6.5053 \\
\hline C19 & 1.7395 & 6.5005 & 6.6821 \\
\hline $\mathrm{C} 20$ & 1.8246 & 7.5067 & 7.7675 \\
\hline $\mathrm{C} 21$ & 3.5389 & 10.7692 & 4.9536 \\
\hline $\mathrm{C} 22$ & 2.9409 & 9.4715 & 8.5217 \\
\hline $\mathrm{C} 23$ & 1.9474 & 10.4584 & 8.6388 \\
\hline $\mathrm{H} 24$ & 1.0315 & 10.3674 & 8.0532 \\
\hline $\mathrm{C} 25$ & 2.1182 & 11.5485 & 9.5004 \\
\hline $\mathrm{C} 26$ & 3.3033 & 11.6313 & 10.2505 \\
\hline $\mathrm{H} 27$ & 3.4458 & 12.4772 & 10.9297 \\
\hline C28 & 4.3052 & 10.657 & 10.1537 \\
\hline $\mathrm{C} 29$ & 4.1139 & 9.5775 & 9.2759 \\
\hline $\mathrm{H} 30$ & 4.8794 & 8.8075 & 9.1876 \\
\hline C31 & 1.0531 & 12.6104 & 9.6327 \\
\hline H32 & 0.6772 & 12.6687 & 10.6676 \\
\hline H33 & 0.1966 & 12.412 & 8.9722 \\
\hline H34 & 1.4523 & 13.607 & 9.3821 \\
\hline C35 & 5.5826 & 10.7694 & 10.9508 \\
\hline H36 & 5.8846 & 9.7959 & 11.3677 \\
\hline H37 & 5.4798 & 11.483 & 11.7812 \\
\hline H38 & 6.4132 & 11.119 & 10.3135 \\
\hline C39 & 5.2647 & 5.8301 & 7.3898 \\
\hline $\mathrm{C} 40$ & 4.6723 & 5.5415 & 8.6323 \\
\hline H41 & 4.3427 & 6.3643 & 9.2692 \\
\hline C42 & 4.5167 & 4.2188 & 9.064 \\
\hline $\mathrm{C} 43$ & 4.9586 & 3.1804 & 8.2252 \\
\hline $\mathrm{H} 44$ & 4.8398 & 2.1432 & 8.553 \\
\hline $\mathrm{C} 45$ & 5.5515 & 3.4402 & 6.9841 \\
\hline C46 & 5.6963 & 4.7774 & 6.5738 \\
\hline $\mathrm{H} 47$ & 6.163 & 5.0004 & 5.6125 \\
\hline C48 & 3.8969 & 3.9045 & 10.4046 \\
\hline $\mathrm{H} 49$ & 4.6163 & 3.3902 & 11.0635 \\
\hline
\end{tabular}




\begin{tabular}{|c|c|c|c|}
\hline H50 & 3.0299 & 3.2324 & 10.2951 \\
\hline H51 & 3.5591 & 4.8152 & 10.92 \\
\hline C52 & 6.0387 & 2.3193 & 6.0973 \\
\hline $\mathrm{H} 53$ & 5.5698 & 2.3649 & 5.1005 \\
\hline H54 & 5.8171 & 1.334 & 6.5321 \\
\hline H55 & 7.1284 & 2.382 & 5.9396 \\
\hline C56 & 1.5842 & 9.4597 & 4.479 \\
\hline C57 & 0.5057 & 10.1287 & 5.0882 \\
\hline H58 & 0.7027 & 10.7916 & 5.9322 \\
\hline C59 & -0.8042 & 9.9675 & 4.6217 \\
\hline C60 & -1.028 & 9.1164 & 3.5259 \\
\hline H61 & -2.0479 & 8.983 & 3.1527 \\
\hline C62 & 0.0253 & 8.4415 & 2.8959 \\
\hline C63 & 1.3303 & 8.6227 & 3.3835 \\
\hline H64 & 2.1632 & 8.1221 & 2.8898 \\
\hline C65 & -1.9567 & 10.6981 & 5.2688 \\
\hline H66 & -1.6332 & 11.2674 & 6.1523 \\
\hline H67 & -2.7469 & 9.9974 & 5.5845 \\
\hline H68 & -2.423 & 11.4063 & 4.5634 \\
\hline C69 & -0.2274 & 7.5206 & 1.7259 \\
\hline $\mathrm{H} 70$ & -1.219 & 7.6925 & 1.2821 \\
\hline H71 & -0.1838 & 6.463 & 2.0394 \\
\hline $\mathrm{H} 72$ & 0.5301 & 7.6534 & 0.9375 \\
\hline $\mathrm{C} 73$ & 5.6347 & 7.9105 & 3.4409 \\
\hline $\mathrm{C} 74$ & 5.3579 & 8.916 & 2.5106 \\
\hline H75 & 4.3255 & 9.2302 & 2.3487 \\
\hline C76 & 6.3982 & 9.5165 & 1.7804 \\
\hline C77 & 7.7121 & 9.0919 & 2.0116 \\
\hline $\mathrm{H} 78$ & 8.5281 & 9.5535 & 1.4476 \\
\hline C79 & 8.011 & 8.0856 & 2.9465 \\
\hline $\mathrm{C} 80$ & 6.9585 & 7.5001 & 3.6605 \\
\hline H81 & 7.165 & 6.7156 & 4.3898 \\
\hline C82 & 6.0874 & 10.5932 & 0.7686 \\
\hline H83 & 5.5752 & 11.4475 & 1.2414 \\
\hline H84 & 5.4162 & 10.2163 & -0.0209 \\
\hline H85 & 7.0004 & 10.9699 & 0.2858 \\
\hline C86 & 9.4402 & 7.6584 & 3.1788 \\
\hline H87 & 9.4994 & 6.7698 & 3.824 \\
\hline H88 & 10.0192 & 8.4638 & 3.6617 \\
\hline H89 & 9.9476 & 7.4268 & 2.2286 \\
\hline $\mathrm{C90}$ & 5.5433 & 13.2495 & 5.3951 \\
\hline H91 & 4.5835 & 13.6422 & 5.7589 \\
\hline H92 & 5.5625 & 13.3833 & 4.3015 \\
\hline H93 & 6.3582 & 13.8477 & 5.822 \\
\hline
\end{tabular}




$\begin{array}{lrrr}\text { C94 } & 8.6776 & 10.2257 & 7.3813 \\ \text { H95 } & 9.1779 & 11.1931 & 7.2493 \\ \text { H96 } & 9.2764 & 9.4484 & 6.8844 \\ \text { H97 } & 8.6618 & 9.9909 & 8.457 \\ \text { C98 } & 7.662 & 7.3575 & 7.898 \\ \text { H99 } & 7.8918 & 7.9461 & 8.7983 \\ \text { H100 } & 8.5535 & 7.3889 & 7.2538 \\ \text { H101 } & 7.5002 & 6.3175 & 8.2025 \\ \text { C102 } & 0.9301 & 7.4458 & 8.9723 \\ \text { H103 } & 1.2837 & 8.1211 & 9.7585 \\ \text { H104 } & 0.9046 & 6.4244 & 9.3743 \\ \text { H105 } & -0.1033 & 7.7277 & 8.7202 \\ \text { C106 } & -0.383 & 5.1679 & 7.3693 \\ \text { H107 } & -0.9847 & 4.3931 & 6.8786 \\ \text { H108 } & -1.0027 & 6.0633 & 7.5144 \\ \text { H109 } & -0.0946 & 4.7949 & 8.3643 \\ \text { C110 } & 4.3041 & 5.5103 & 2.5487 \\ \text { H111 } & 5.1111 & 6.0136 & 2.006 \\ \text { H112 } & 3.4287 & 5.442 & 1.8868 \\ \text { H113 } & 4.6266 & 4.4838 & 2.7749 \\ \text { C114 } & 2.9874 & 11.969 & 4.2303 \\ \text { H115 } & 3.7553 & 12.4283 & 3.5942 \\ \text { H116 } & 2.636 & 12.7437 & 4.9305 \\ \text { H117 } & 2.1403 & 11.6887 & 3.594 \\ \text { C118 } & 2.0606 & 3.6392 & 3.5552 \\ \text { H119 } & 1.2974 & 2.8864 & 3.7867 \\ \text { H120 } & 3.0456 & 3.151 & 3.5385 \\ \text { H121 } & 1.8647 & 4.0302 & 2.5456\end{array}$

\section{Table S7. Selected Kohn-Sham Orbital Energies for Complex 2 in THF}

\begin{tabular}{|c|c|c|c|c|c|c|c|}
\hline \multicolumn{4}{|c|}{ Alpha Orbitals } & \multicolumn{4}{|c|}{ Beta Orbitals } \\
\hline Number & Occ. & $\begin{array}{c}\text { Energy } \\
(\text { Hartree })\end{array}$ & $\begin{array}{c}\text { Energy } \\
(\mathrm{eV})\end{array}$ & Number & Occ. & \begin{tabular}{|c|} 
Energy \\
(Hartree)
\end{tabular} & $\begin{array}{l}\text { Energy } \\
(\mathrm{eV})\end{array}$ \\
\hline 235 & 0 & -0.02729 & -0.7425 & 235 & 0 & -0.02651 & -0.72142 \\
\hline 234 & 0 & -0.02949 & -0.80255 & 234 & 0 & -0.02775 & -0.75501 \\
\hline 233 & 0 & -0.03537 & -0.96257 & 233 & 0 & -0.03257 & -0.88629 \\
\hline 232 & 0 & -0.04039 & -1.09905 & 232 & 0 & -0.03954 & -1.07591 \\
\hline 231 & 0 & -0.04517 & -1.22901 & 231 & 0 & -0.04432 & -1.20603 \\
\hline 230 & 0 & -0.08972 & -2.44143 & 230 & 0 & -0.07978 & -2.17099 \\
\hline 229 & 0 & -0.1004 & -2.73191 & 229 & 0 & -0.08145 & -2.21632 \\
\hline 228 & 0 & -0.11035 & -3.00284 & 228 & 0 & -0.10019 & -2.72632 \\
\hline 227 & 1 & -0.14749 & -4.01331 & 227 & 0 & -0.10967 & -2.98439 \\
\hline
\end{tabular}




$\begin{array}{llllllll}226 & 1 & -0.22355 & -6.08298 & 226 & 1 & -0.22089 & -6.0107 \\ 225 & 1 & -0.23134 & -6.29504 & 225 & 1 & -0.22832 & -6.21295 \\ 224 & 1 & -0.24141 & -6.56899 & 224 & 1 & -0.22923 & -6.23763\end{array}$

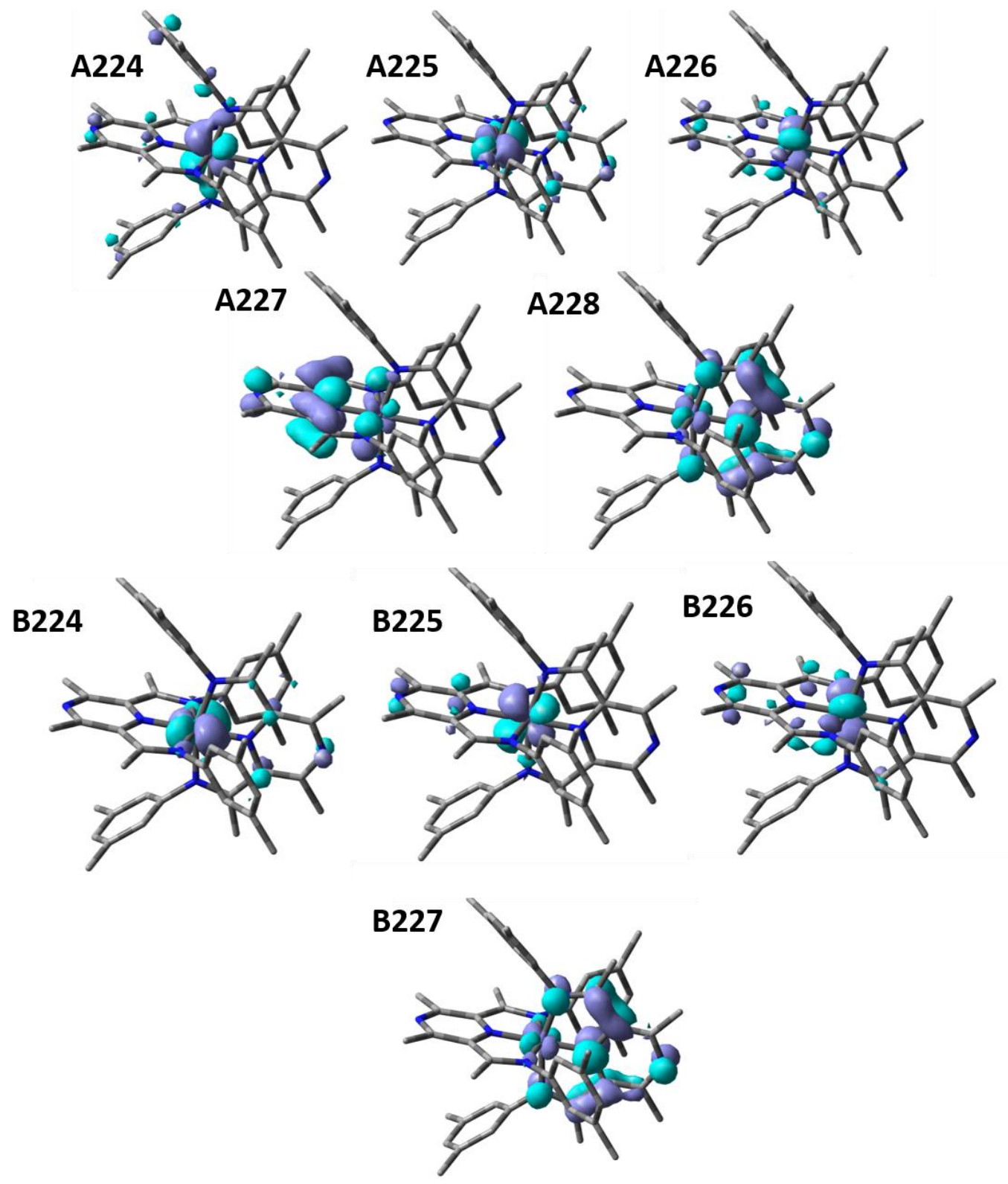

Figure S26. Visualization of selected orbitals from complex $\mathbf{2}$ in THF.

Table S8. Mulliken Charges and Spin Densities for Complex 2 (THF solvation)

\begin{tabular}{c|c|c|c} 
Number $^{\mathrm{a}}$ & Atom & $\begin{array}{c}\text { Mulliken } \\
\text { Charges }^{\mathrm{b}}\end{array}$ & $\begin{array}{r}\text { Mulliken Spin } \\
\text { Densities }^{\mathrm{b}}\end{array}$ \\
\hline 1 & $\mathrm{Fe}$ & 0.318057 & 0.049302 \\
2 & $\mathrm{~N}$ & -0.24474 & 0.369382
\end{tabular}




\begin{tabular}{|c|c|c|c|}
\hline 3 & $\mathrm{~N}$ & -0.22765 & 0.112187 \\
\hline 4 & $\mathrm{~N}$ & -0.17978 & 0.013054 \\
\hline 5 & $\mathrm{~N}$ & -0.17744 & -0.00051 \\
\hline 6 & $\mathrm{~N}$ & -0.19143 & 0.00764 \\
\hline 7 & $\mathrm{~N}$ & -0.23594 & 0.103594 \\
\hline 8 & $\mathrm{~N}$ & -0.12188 & 0.107234 \\
\hline 9 & $\mathrm{~N}$ & -0.07766 & -0.0005 \\
\hline 10 & C & 0.173467 & 0.06376 \\
\hline 11 & C & -0.17991 & -0.04368 \\
\hline 12 & $C$ & -0.18037 & -0.03344 \\
\hline 13 & $C$ & 0.170189 & 0.054355 \\
\hline 14 & C & -0.07186 & 0.089882 \\
\hline 15 & C & -0.05443 & -0.00128 \\
\hline 16 & $C$ & 0.15879 & -0.0001 \\
\hline 17 & $C$ & -0.15678 & -0.00045 \\
\hline 18 & $\mathrm{C}$ & -0.16056 & -0.00071 \\
\hline 19 & C & 0.165116 & 0.000186 \\
\hline 20 & $C$ & -0.04704 & -0.00193 \\
\hline 21 & $C$ & -0.06758 & 0.100946 \\
\hline 22 & $C$ & 0.131606 & -0.00225 \\
\hline 23 & $C$ & -0.07365 & 0.001572 \\
\hline 25 & C & -0.04814 & -0.00094 \\
\hline 26 & C & -0.08263 & 0.001819 \\
\hline 28 & C & -0.04914 & -0.00124 \\
\hline 29 & C & -0.04311 & 0.003186 \\
\hline 31 & C & 0.163476 & 0.000027 \\
\hline 35 & $C$ & 0.162295 & 0.000171 \\
\hline 39 & $C$ & 0.132512 & -0.0056 \\
\hline 40 & C & -0.06664 & 0.00374 \\
\hline 42 & C & -0.04091 & 0.000334 \\
\hline 43 & $C$ & -0.09495 & -0.00016 \\
\hline 45 & $C$ & -0.04196 & 0.000312 \\
\hline 46 & C & -0.07504 & 0.003072 \\
\hline 48 & C & 0.15871 & 0.000179 \\
\hline 52 & C & 0.159467 & 0.000129 \\
\hline 56 & $C$ & 0.141041 & -0.01116 \\
\hline 57 & $C$ & -0.08458 & 0.010219 \\
\hline 59 & C & -0.04523 & -0.00479 \\
\hline 60 & C & -0.09464 & 0.009679 \\
\hline 62 & $C$ & -0.04353 & -0.00524 \\
\hline 63 & C & -0.0726 & 0.013987 \\
\hline 65 & C & 0.158422 & 0.000227 \\
\hline 69 & C & 0.156453 & 0.000201 \\
\hline 73 & C & 0.123948 & 0.001755 \\
\hline
\end{tabular}




\begin{tabular}{rrrr}
74 & $C$ & -0.05069 & -0.00217 \\
76 & $C$ & -0.04672 & 0.001413 \\
77 & $C$ & -0.08603 & -0.00178 \\
79 & $C$ & -0.04528 & 0.001275 \\
80 & $C$ & -0.05503 & -0.00233 \\
82 & $C$ & 0.162013 & 0.000074 \\
86 & $C$ & 0.161568 & 0.000015 \\
90 & $C$ & 0.189489 & -0.00092 \\
94 & $C$ & 0.188159 & -0.00132 \\
98 & $C$ & 0.233571 & -0.00173 \\
102 & $C$ & 0.261751 & 0.000223 \\
106 & $C$ & 0.227623 & 0.000046 \\
110 & $C$ & 0.270159 & 0.000298 \\
114 & $C$ & 0.221687 & -0.00125 \\
118 & $C$ & 0.225971 & $-9 E-06$ \\
\hline
\end{tabular}

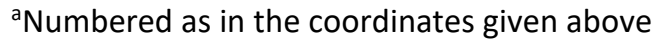

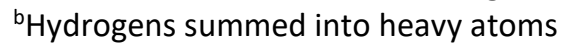

Table S9. Optimized Coordinates for Complex 2 (gas phase, no solvation)

\begin{tabular}{lrrrr} 
Atom & $X$ & $Y$ & $X$ \\
\hline Fe1 & 3.9374 & 8.0984 & 5.9348 \\
N2 & 5.1883 & 9.5009 & 6.127 \\
N3 & 2.9279 & 9.6174 & 5.0083 \\
N4 & 2.7659 & 8.3638 & 7.5899 \\
N5 & 2.7301 & 6.6598 & 5.728 \\
N6 & 4.601 & 7.3337 & 4.2018 \\
N7 & 5.3721 & 7.1456 & 6.9652 \\
N8 & 7.005 & 11.5048 & 6.3996 \\
N9 & 1.0101 & 4.5767 & 5.4231 \\
C10 & 4.8727 & 10.7416 & 5.665 \\
C11 & 5.8034 & 11.7838 & 5.8721 \\
C12 & 7.3454 & 10.2676 & 6.7797 \\
C13 & 6.3954 & 9.2239 & 6.6967 \\
C14 & 6.4624 & 7.8479 & 7.1909 \\
C15 & 4.007 & 6.2248 & 3.8129 \\
C16 & 2.9209 & 5.7929 & 4.693 \\
C17 & 2.047 & 4.6868 & 4.5843 \\
C18 & 0.8031 & 5.4506 & 6.4203 \\
C19 & 1.7236 & 6.501 & 6.6304 \\
C20 & 1.7986 & 7.4828 & 7.718 \\
C21 & 3.5724 & 10.7629 & 4.9858 \\
C22 & 2.9152 & 9.4386 & 8.5135
\end{tabular}




\begin{tabular}{|c|c|c|c|}
\hline $\mathrm{C} 23$ & 1.904 & 10.4069 & 8.6403 \\
\hline $\mathrm{H} 24$ & 0.9917 & 10.3031 & 8.05 \\
\hline $\mathrm{C} 25$ & 2.0505 & 11.4931 & 9.5121 \\
\hline $\mathrm{C} 26$ & 3.2339 & 11.5975 & 10.2597 \\
\hline $\mathrm{H} 27$ & 3.3581 & 12.4403 & 10.9463 \\
\hline $\mathrm{C} 28$ & 4.257 & 10.6458 & 10.1539 \\
\hline C29 & 4.0861 & 9.5683 & 9.2715 \\
\hline H3O & 4.8651 & 8.8117 & 9.1833 \\
\hline C31 & 0.956 & 12.5218 & 9.6655 \\
\hline H32 & 0.5067 & 12.4743 & 10.6717 \\
\hline H33 & 0.1486 & 12.3728 & 8.9336 \\
\hline H34 & 1.3461 & 13.5446 & 9.5392 \\
\hline C35 & 5.5313 & 10.7912 & 10.9507 \\
\hline H36 & 6.0018 & 9.8158 & 11.1468 \\
\hline H37 & 5.3495 & 11.2818 & 11.9187 \\
\hline H38 & 6.2666 & 11.4099 & 10.407 \\
\hline C39 & 5.2403 & 5.8036 & 7.4379 \\
\hline $\mathrm{C} 40$ & 4.6541 & 5.5488 & 8.6847 \\
\hline H41 & 4.3283 & 6.3866 & 9.3039 \\
\hline $\mathrm{C} 42$ & 4.4942 & 4.2327 & 9.1452 \\
\hline $\mathrm{C} 43$ & 4.9239 & 3.179 & 8.3266 \\
\hline $\mathrm{H} 44$ & 4.8009 & 2.1491 & 8.6757 \\
\hline C45 & 5.5134 & 3.409 & 7.0747 \\
\hline C46 & 5.6633 & 4.7328 & 6.6374 \\
\hline $\mathrm{H} 47$ & 6.1225 & 4.939 & 5.6686 \\
\hline $\mathrm{C} 48$ & 3.8998 & 3.9547 & 10.505 \\
\hline $\mathrm{H} 49$ & 4.6844 & 3.6725 & 11.2278 \\
\hline $\mathrm{H} 50$ & 3.1843 & 3.1184 & 10.4683 \\
\hline H51 & 3.3797 & 4.8345 & 10.9118 \\
\hline C52 & 5.9787 & 2.2506 & 6.2253 \\
\hline $\mathrm{H} 53$ & 5.1481 & 1.5613 & 6.0006 \\
\hline H54 & 6.7494 & 1.6595 & 6.7468 \\
\hline H55 & 6.4071 & 2.5892 & 5.2705 \\
\hline C56 & 1.611 & 9.4987 & 4.4774 \\
\hline C57 & 0.5539 & 10.238 & 5.0367 \\
\hline H58 & 0.7629 & 10.9286 & 5.8557 \\
\hline C59 & -0.7545 & 10.1028 & 4.5562 \\
\hline C60 & -0.9916 & 9.2087 & 3.5002 \\
\hline H61 & -2.0095 & 9.0971 & 3.1147 \\
\hline C62 & 0.0433 & 8.4609 & 2.9234 \\
\hline C63 & 1.345 & 8.6134 & 3.4251 \\
\hline H64 & 2.1654 & 8.0492 & 2.9828 \\
\hline C65 & -1.8873 & 10.9108 & 5.1424 \\
\hline H66 & -1.5679 & 11.4828 & 6.0259 \\
\hline
\end{tabular}




\begin{tabular}{|c|c|c|c|}
\hline $\mathrm{H} 67$ & -2.7284 & 10.2644 & 5.4408 \\
\hline H68 & -2.2837 & 11.6292 & 4.4054 \\
\hline C69 & -0.2382 & 7.4869 & 1.8046 \\
\hline $\mathrm{H} 70$ & -0.4802 & 6.487 & 2.2056 \\
\hline H71 & 0.6297 & 7.3726 & 1.1372 \\
\hline $\mathrm{H} 72$ & -1.0961 & 7.8073 & 1.195 \\
\hline C73 & 5.6337 & 7.9382 & 3.421 \\
\hline $\mathrm{C} 74$ & 5.3167 & 8.9126 & 2.4655 \\
\hline H75 & 4.2715 & 9.1823 & 2.303 \\
\hline C76 & 6.326 & 9.5324 & 1.7124 \\
\hline C77 & 7.6582 & 9.1635 & 1.9448 \\
\hline $\mathrm{H} 78$ & 8.4526 & 9.643 & 1.3646 \\
\hline C79 & 8.0005 & 8.1915 & 2.8967 \\
\hline $\mathrm{C} 80$ & 6.974 & 7.5854 & 3.635 \\
\hline H81 & 7.2135 & 6.8228 & 4.3785 \\
\hline C82 & 5.9851 & 10.5517 & 0.652 \\
\hline H83 & 6.688 & 11.3991 & 0.6668 \\
\hline H84 & 4.9663 & 10.9475 & 0.7775 \\
\hline H85 & 6.0426 & 10.1058 & -0.3558 \\
\hline C86 & 9.4455 & 7.8115 & 3.1141 \\
\hline H87 & 9.5512 & 7.019 & 3.8695 \\
\hline H88 & 10.0401 & 8.6789 & 3.4459 \\
\hline H89 & 9.9067 & 7.4489 & 2.181 \\
\hline $\mathrm{C90}$ & 5.5719 & 13.2413 & 5.5674 \\
\hline H91 & 4.594 & 13.5969 & 5.9199 \\
\hline H92 & 5.6345 & 13.4475 & 4.4864 \\
\hline H93 & 6.3641 & 13.8182 & 6.0607 \\
\hline C94 & 8.7622 & 10.1203 & 7.272 \\
\hline H95 & 9.285 & 11.0661 & 7.0843 \\
\hline H96 & 9.2983 & 9.3077 & 6.761 \\
\hline H97 & 8.8048 & 9.9171 & 8.3536 \\
\hline C98 & 7.647 & 7.3059 & 7.9424 \\
\hline H99 & 7.8865 & 7.9332 & 8.8137 \\
\hline $\mathrm{H} 100$ & 8.5436 & 7.2872 & 7.3046 \\
\hline H101 & 7.4588 & 6.2855 & 8.2942 \\
\hline C102 & 0.8966 & 7.413 & 8.9206 \\
\hline H103 & 1.2826 & 8.0335 & 9.7374 \\
\hline H104 & 0.8106 & 6.3782 & 9.2775 \\
\hline H105 & -0.1215 & 7.7629 & 8.6881 \\
\hline C106 & -0.4502 & 5.2079 & 7.2209 \\
\hline $\mathrm{H} 107$ & -1.0665 & 4.4859 & 6.6707 \\
\hline H108 & -1.0309 & 6.1257 & 7.386 \\
\hline H109 & -0.2263 & 4.7702 & 8.2074 \\
\hline C110 & 4.3712 & 5.4997 & 2.5464 \\
\hline
\end{tabular}




$\begin{array}{lrrr}\mathrm{H} 111 & 5.1585 & 6.0302 & 1.9997 \\ \mathrm{H} 112 & 3.5002 & 5.3951 & 1.8827 \\ \mathrm{H} 113 & 4.7321 & 4.4833 & 2.7644 \\ \mathrm{C} 114 & 3.0777 & 11.9767 & 4.2463 \\ \mathrm{H} 115 & 3.8867 & 12.4208 & 3.6515 \\ \mathrm{H} 116 & 2.7148 & 12.7556 & 4.9354 \\ \mathrm{H} 117 & 2.2518 & 11.7171 & 3.5743 \\ \mathrm{C} 118 & 2.172 & 3.5727 & 3.5771 \\ \mathrm{H} 119 & 1.4343 & 2.8021 & 3.8323 \\ \mathrm{H} 120 & 3.1748 & 3.1217 & 3.5775 \\ \mathrm{H} 121 & 1.9637 & 3.9172 & 2.552\end{array}$

Table S10. Energies of selected Kohn-Sham orbitals for complex $\mathbf{2}$ in the gas phase

\begin{tabular}{|l|l|l|l|l|l|l|l|}
\hline \multicolumn{5}{|c|}{ Alpha Orbitals } & \multicolumn{5}{c|}{ Beta Orbitals } \\
\hline Number & Occ. & $\begin{array}{c}\text { Energy } \\
\text { (Hartree) })\end{array}$ & $\begin{array}{c}\text { Energy } \\
(\mathrm{eV})\end{array}$ & Number & Occ. & $\begin{array}{c}\text { Energy } \\
\text { (Hartree) }\end{array}$ & $\begin{array}{l}\text { Energy } \\
(\mathrm{eV})\end{array}$ \\
\hline 235 & 0 & -0.09318 & -2.53566 & 235 & 0 & -0.09244 & -2.51546 \\
234 & 0 & -0.09728 & -2.64717 & 234 & 0 & -0.09533 & -2.59413 \\
233 & 0 & -0.10538 & -2.86764 & 233 & 0 & -0.10011 & -2.72421 \\
232 & 0 & -0.10696 & -2.9105 & 232 & 0 & -0.103 & -2.8028 \\
231 & 0 & -0.11286 & -3.07111 & 231 & 0 & -0.11212 & -3.05088 \\
230 & 0 & -0.16412 & -4.46586 & 230 & 0 & -0.1583 & -4.30756 \\
229 & 0 & -0.16493 & -4.48802 & 229 & 0 & -0.15979 & -4.34797 \\
228 & 0 & -0.17905 & -4.87231 & 228 & 0 & -0.16404 & -4.46382 \\
227 & 1 & -0.21529 & -5.85834 & 227 & 0 & -0.16649 & -4.53051 \\
226 & 1 & -0.29086 & -7.91478 & 226 & 1 & -0.29304 & -7.97398 \\
225 & 1 & -0.29695 & -8.08054 & 225 & 1 & -0.29853 & -8.12343 \\
224 & 1 & -0.30843 & -8.39272 & 224 & 1 & -0.30393 & -8.27028 \\
223 & 1 & -0.32217 & -8.76678 & 223 & 1 & -0.31839 & -8.66386
\end{tabular}



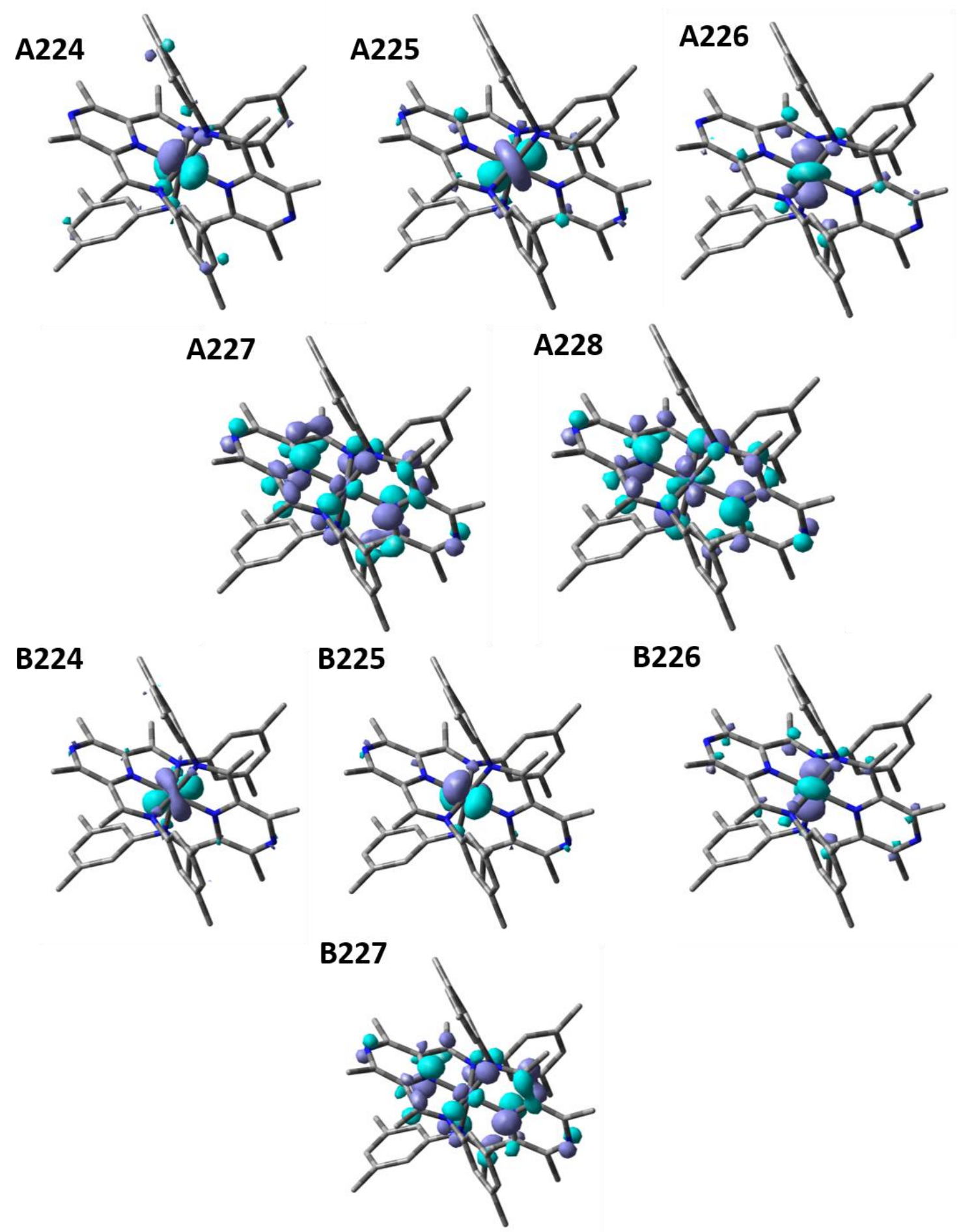

Figure S27. Visualizations of selected orbitals of complex 2, calculated in the gas phase. 


\section{Supplementary Computational Results for Complex 3}

Table S11. Optimized Coordinates for Complex 3

\begin{tabular}{lrrr} 
Atom & $\mathrm{X}$ & $\mathrm{Y}$ & $\mathrm{Z}$ \\
\hline Fe1 & -0.0201 & -0.1345 & -0.0016 \\
N2 & -0.2736 & -1.512 & -1.4544 \\
N3 & 1.7858 & -0.4454 & -0.5086 \\
N4 & 0.9546 & 1.1911 & 1.23 \\
N5 & -0.1756 & -1.5246 & 1.4533 \\
N6 & -1.839 & 0.0901 & 0.5043 \\
N7 & -0.5695 & 1.4151 & -1.2342 \\
N8 & 4.38 & -0.9011 & -1.2481 \\
N9 & -4.4535 & 0.4066 & 1.2449 \\
C10 & 0.8391 & -1.9926 & -1.9903 \\
C11 & 2.055 & -1.3942 & -1.4661 \\
C12 & 3.397 & -1.626 & -1.8147 \\
C13 & 4.1162 & 0.0197 & -0.3056 \\
C14 & 2.791 & 0.2537 & 0.1187 \\
C15 & 2.282 & 1.1652 & 1.1229 \\
C16 & -1.381 & -1.6638 & 1.9873 \\
C17 & -2.3717 & -0.7409 & 1.4613 \\
C18 & -3.7238 & -0.5737 & 1.8092 \\
C19 & -3.9334 & 1.2132 & 0.3037 \\
C20 & -2.5981 & 1.0516 & -0.1213 \\
C21 & -1.8462 & 1.7771 & -1.1253 \\
C22 & -1.5547 & -1.9317 & -1.9026 \\
C23 & -2.237 & -2.9696 & -1.2468 \\
H24 & -1.754 & -3.4691 & -0.4048 \\
C25 & -3.5152 & -3.3638 & -1.6597 \\
C26 & -4.1064 & -2.7011 & -2.7475 \\
H27 & -5.105 & -3.0029 & -3.0785 \\
C28 & -3.4486 & -1.6623 & -3.4178 \\
C29 & -2.17 & -1.2823 & -2.981 \\
H30 & -1.6369 & -0.4707 & -3.481 \\
C31 & 0.3349 & 2.1411 & -2.0422 \\
C32 & 1.0903 & 1.4875 & -3.0274 \\
H33 & 0.9352 & 0.4212 & -3.1882 \\
C34 & 2.0334 & 2.18 & -3.8006 \\
C35 & 2.2123 & 3.5517 & -3.5812 \\
& 2.9474 & 4.0994 & -4.1793 \\
H36 & 1.4704 & 4.2369 & -2.6049 \\
0.5413 & 3.5219 & -1.8426 \\
C39 & & & -1.0659
\end{tabular}




\begin{tabular}{|c|c|c|c|}
\hline C40 & 0.9309 & -2.289 & 1.9104 \\
\hline C41 & 1.3044 & -3.4718 & 1.2522 \\
\hline $\mathrm{H} 42$ & 0.7113 & -3.8119 & 0.4011 \\
\hline $\mathrm{C} 43$ & 2.4179 & -4.2089 & 1.6733 \\
\hline C44 & 3.1573 & -3.7442 & 2.7725 \\
\hline $\mathrm{H} 45$ & 4.029 & -4.3138 & 3.1094 \\
\hline C46 & 2.8067 & -2.5674 & 3.4466 \\
\hline C47 & 1.6906 & -1.8434 & 3.0009 \\
\hline $\mathrm{H} 48$ & 1.3997 & -0.9176 & 3.5022 \\
\hline C49 & 0.2997 & 2.151 & 2.0342 \\
\hline C50 & 0.5132 & 3.5316 & 1.8397 \\
\hline H51 & 1.2179 & 3.8517 & 1.07 \\
\hline C52 & -0.1696 & 4.4881 & 2.5977 \\
\hline C53 & -1.0907 & 4.0515 & 3.5641 \\
\hline H54 & -1.6366 & 4.7911 & 4.1582 \\
\hline C55 & -1.3283 & 2.6879 & 3.7781 \\
\hline C56 & -0.6247 & 1.7488 & 3.0101 \\
\hline H57 & -0.7939 & 0.6841 & 3.1672 \\
\hline C58 & -4.4815 & -1.4154 & 2.8089 \\
\hline H59 & -4.4884 & -2.4825 & 2.5381 \\
\hline $\mathrm{H} 60$ & -5.5172 & -1.0536 & 2.8314 \\
\hline H61 & -4.0652 & -1.3386 & 3.8255 \\
\hline C62 & -1.682 & -2.6881 & 3.052 \\
\hline $\mathrm{H} 63$ & -2.4835 & -3.3717 & 2.7335 \\
\hline H64 & -2.0184 & -2.215 & 3.9875 \\
\hline H65 & -0.7943 & -3.2896 & 3.2796 \\
\hline C66 & 3.5927 & -2.0924 & 4.6462 \\
\hline $\mathrm{H} 67$ & 3.0477 & -2.2932 & 5.5853 \\
\hline $\mathrm{H} 68$ & 3.7714 & -1.006 & 4.6069 \\
\hline H69 & 4.5682 & -2.5962 & 4.7172 \\
\hline C70 & 2.838 & -5.4608 & 0.94 \\
\hline H71 & 1.9979 & -5.9106 & 0.3893 \\
\hline $\mathrm{H} 72$ & 3.2387 & -6.2197 & 1.6303 \\
\hline H73 & 3.6331 & -5.2402 & 0.206 \\
\hline C74 & 5.3375 & 0.7533 & 0.196 \\
\hline H75 & 5.5916 & 0.4728 & 1.2312 \\
\hline H76 & 5.2157 & 1.8456 & 0.1698 \\
\hline $\mathrm{H} 77$ & 6.1815 & 0.4721 & -0.4465 \\
\hline $\mathrm{C} 78$ & 3.8782 & -2.6488 & -2.8169 \\
\hline H79 & 4.9743 & -2.6031 & -2.8385 \\
\hline $\mathrm{H} 80$ & 3.5032 & -2.4513 & -3.8334 \\
\hline H81 & 3.575 & -3.6728 & -2.5499 \\
\hline C82 & 0.8308 & -3.0598 & -3.0552 \\
\hline H83 & 1.3968 & -3.9479 & -2.7355 \\
\hline
\end{tabular}




\begin{tabular}{lrrr} 
H84 & 1.2939 & -2.7048 & -3.9888 \\
H85 & -0.1931 & -3.3757 & -3.2862 \\
C86 & 1.6677 & 5.7208 & -2.3978 \\
H87 & 1.163 & 6.0745 & -1.4862 \\
H88 & 1.2631 & 6.2999 & -3.2461 \\
H89 & 2.7364 & 5.9784 & -2.3166 \\
C90 & 2.8636 & 1.441 & -4.8238 \\
H91 & 2.2529 & 0.7294 & -5.4024 \\
H92 & 3.6625 & 0.8582 & -4.3329 \\
H93 & 3.3446 & 2.1313 & -5.533 \\
C94 & -4.0838 & -0.9701 & -4.6008 \\
H95 & -4.0197 & 0.1261 & -4.5074 \\
H96 & -5.1449 & -1.2406 & -4.7072 \\
H97 & -3.5754 & -1.2405 & -5.5424 \\
C98 & -4.2607 & -4.4577 & -0.9323 \\
H99 & -3.5782 & -5.1023 & -0.3578 \\
H100 & -4.826 & -5.0956 & -1.63 \\
H101 & -4.9909 & -4.0327 & -0.2212 \\
C102 & -4.8882 & 2.2722 & -0.1945 \\
H103 & -5.2134 & 2.0814 & -1.2303 \\
H104 & -4.4533 & 3.2815 & -0.165 \\
H105 & -5.7774 & 2.247 & 0.4481 \\
C106 & -2.482 & 2.7981 & -2.0376 \\
H107 & -2.6721 & 3.7593 & -1.5325 \\
H108 & -3.4472 & 2.4338 & -2.4137 \\
H109 & -1.8362 & 3.0041 & -2.8997 \\
C110 & -2.3511 & 2.2254 & 4.7892 \\
H111 & -1.984 & 1.3649 & 5.3713 \\
H112 & -3.2804 & 1.9042 & 4.287 \\
H113 & -2.6155 & 3.0262 & 5.4962 \\
C114 & 0.0842 & 5.9643 & 2.3966 \\
H115 & 0.6743 & 6.155 & 1.4875 \\
H116 & 0.6403 & 6.3937 & 3.2481 \\
H117 & -0.8591 & 6.5286 & 2.3145 \\
C118 & 3.1865 & 1.9536 & 2.0391 \\
H119 & 3.6491 & 2.8201 & 1.5387 \\
& 4.003 & 1.3223 & 2.4142 \\
\hline 120 & 2.6273 & 2.3352 & 2.9019
\end{tabular}


Table S12. Energies of selected orbitals for complex 3

\begin{tabular}{|c|c|c|c|c|c|c|c|}
\hline \multicolumn{5}{|c|}{ Alpha Orbitals } & \multicolumn{4}{c|}{ Beta Orbitals } \\
\hline Number & Occ. & $\begin{array}{c}\text { Energy } \\
\text { (Hartree) })\end{array}$ & $\begin{array}{c}\text { Energy } \\
(\mathrm{eV})\end{array}$ & $\begin{array}{c}\text { Number } \\
\text { Occ. }\end{array}$ & $\begin{array}{c}\text { Energy } \\
\text { (Hartree) }\end{array}$ & $\begin{array}{l}\text { Energy } \\
(\mathrm{eV})\end{array}$ \\
\hline 235 & 0 & -0.00113 & -0.03062 & 235 & 0 & -0.00054 & -0.01466 \\
234 & 0 & -0.00528 & -0.1436 & 234 & 0 & -0.00356 & -0.09691 \\
233 & 0 & -0.00653 & -0.17762 & 233 & 0 & -0.00412 & -0.11214 \\
232 & 0 & -0.00871 & -0.23704 & 232 & 0 & -0.00562 & -0.15292 \\
231 & 0 & -0.01188 & -0.32319 & 231 & 0 & -0.00723 & -0.1968 \\
230 & 0 & -0.06088 & -1.65667 & 230 & 0 & -0.04504 & -1.22563 \\
229 & 0 & -0.06091 & -1.65746 & 229 & 0 & -0.0492 & -1.33883 \\
228 & 1 & -0.11267 & -3.06594 & 228 & 0 & -0.05099 & -1.38748 \\
227 & 1 & -0.11418 & -3.10706 & 227 & 0 & -0.05142 & -1.39929 \\
226 & 1 & -0.178 & -4.84373 & 226 & 1 & -0.16795 & -4.57022 \\
225 & 1 & -0.1976 & -5.37686 & 225 & 1 & -0.17348 & -4.72071 \\
224 & 1 & -0.19794 & -5.38619 & 224 & 1 & -0.17557 & -4.77753 \\
223 & 1 & -0.21901 & -5.95946 & 223 & 1 & -0.21212 & -5.77211 \\
\end{tabular}



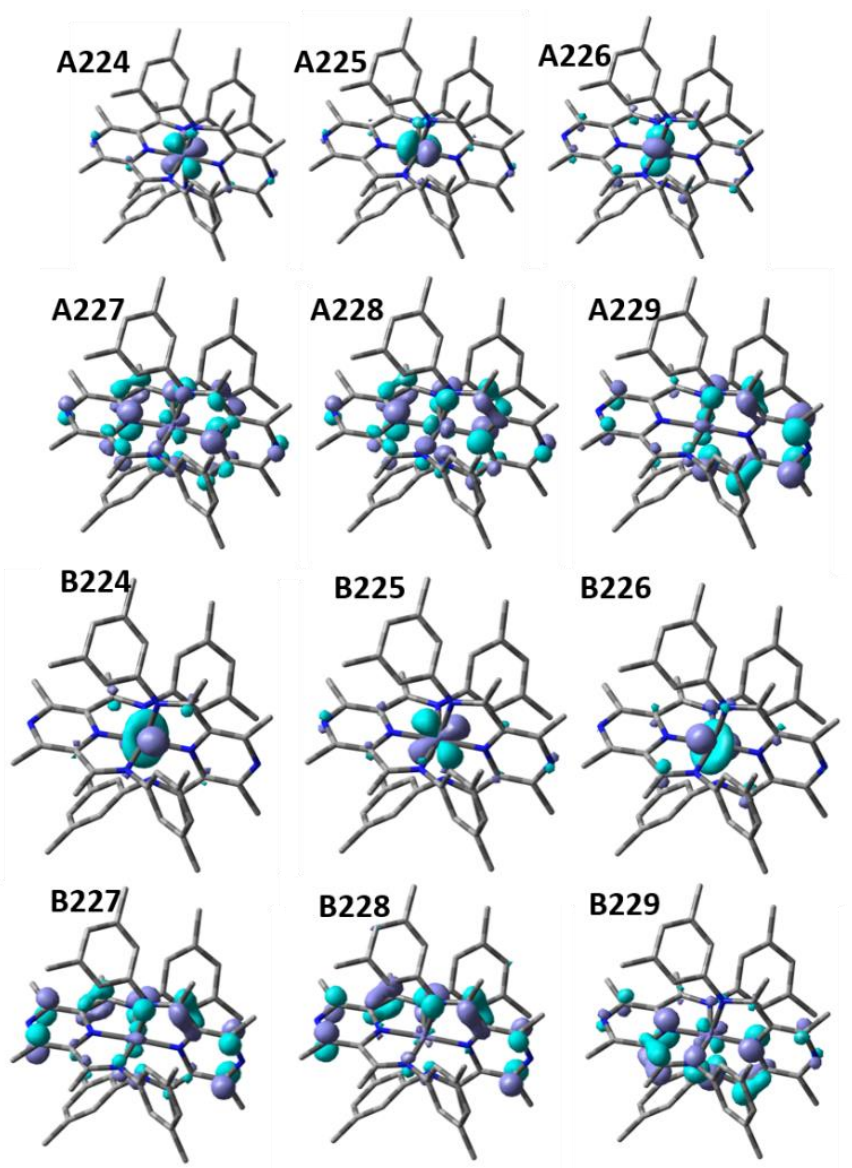

Figure S28. Visualizations of selected orbitals for complex 3.

Table S13. Mulliken Charges and Spin Densities for Complex 3.

\begin{tabular}{c|rrr} 
Number & Atom & $\begin{array}{r}\text { Mulliken } \\
\text { Charges }^{\mathrm{b}}\end{array}$ & $\begin{array}{r}\text { Mulliken Spin } \\
\text { Densities }^{\mathrm{b}}\end{array}$ \\
\hline 1 & $\mathrm{Fe}$ & 0.351029 & 0.198428 \\
2 & $\mathrm{~N}$ & -0.22916 & 0.113134 \\
3 & $\mathrm{~N}$ & -0.252 & 0.356012 \\
4 & $\mathrm{~N}$ & -0.21293 & 0.128646 \\
5 & $\mathrm{~N}$ & -0.22943 & 0.115624 \\
6 & $\mathrm{~N}$ & -0.25222 & 0.356433 \\
7 & $\mathrm{~N}$ & -0.21297 & 0.1265 \\
8 & $\mathrm{~N}$ & -0.10596 & 0.089152 \\
9 & $\mathrm{~N}$ & -0.10639 & 0.089431 \\
10 & $\mathrm{C}$ & -0.09112 & 0.074539 \\
11 & $\mathrm{C}$ & 0.169617 & 0.03017 \\
12 & $\mathrm{C}$ & -0.18866 & -0.02314 \\
13 & $\mathrm{C}$ & -0.18319 & -0.04088 \\
14 & $\mathrm{C}$ & 0.188013 & 0.046616 \\
15 & $\mathrm{C}$ & -0.08324 & 0.104183
\end{tabular}




\begin{tabular}{|c|c|c|c|}
\hline 16 & C & -0.09101 & 0.075841 \\
\hline 17 & C & 0.169246 & 0.031971 \\
\hline 18 & C & -0.18829 & -0.0251 \\
\hline 19 & $C$ & -0.18342 & -0.03935 \\
\hline 20 & C & 0.18854 & 0.04521 \\
\hline 21 & C & -0.08365 & 0.102261 \\
\hline 22 & C & 0.132928 & -0.00412 \\
\hline 23 & C & -0.06924 & 0.001622 \\
\hline 25 & C & -0.02868 & 0.001465 \\
\hline 26 & C & -0.10147 & -0.00241 \\
\hline 28 & C & -0.03333 & 0.001935 \\
\hline 29 & C & -0.06976 & 0.000437 \\
\hline 31 & C & 0.148101 & -0.01352 \\
\hline 32 & C & -0.06971 & 0.023929 \\
\hline 34 & C & -0.02873 & -0.00979 \\
\hline 35 & C & -0.11281 & 0.018134 \\
\hline 37 & C & -0.03412 & -0.0086 \\
\hline 38 & C & -0.09756 & 0.016189 \\
\hline 40 & C & 0.132651 & -0.00441 \\
\hline 41 & C & -0.06922 & 0.001979 \\
\hline 43 & C & -0.02909 & 0.001307 \\
\hline 44 & C & -0.10125 & -0.00222 \\
\hline 46 & C & -0.03394 & 0.00185 \\
\hline 47 & C & -0.06925 & 0.000658 \\
\hline 49 & $C$ & 0.148766 & -0.01397 \\
\hline 50 & C & -0.09877 & 0.016904 \\
\hline 52 & C & -0.0341 & -0.00903 \\
\hline 53 & C & -0.11336 & 0.018975 \\
\hline 55 & C & -0.02871 & -0.01023 \\
\hline 56 & C & -0.06963 & 0.024846 \\
\hline 58 & C & 0.152353 & -0.00125 \\
\hline 62 & C & 0.187956 & -0.00171 \\
\hline 66 & C & 0.129405 & 0.000193 \\
\hline 70 & C & 0.127168 & 0.00022 \\
\hline 74 & C & 0.157674 & -0.00061 \\
\hline 78 & C & 0.151938 & -0.00128 \\
\hline 82 & C & 0.188533 & -0.00167 \\
\hline 86 & C & 0.123844 & 0.000416 \\
\hline 90 & C & 0.128078 & 0.00039 \\
\hline 94 & C & 0.129635 & 0.000186 \\
\hline 98 & C & 0.127224 & 0.00021 \\
\hline 102 & $C$ & 0.15749 & -0.00065 \\
\hline 106 & C & 0.172662 & -0.00144 \\
\hline 110 & C & 0.127999 & 0.000418 \\
\hline
\end{tabular}




\begin{tabular}{llll}
114 & $C$ & 0.123559 & 0.000425 \\
118 & $C$ & 0.171923 & -0.00147 \\
\hline
\end{tabular}

${ }^{a}$ Numbered as in the coordinates given above

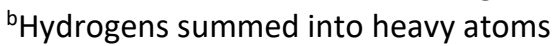

\section{TD-DFT Results on Complexes 1-3}

Methods: Time-dependent DFT using the Tamm-Dancoff approximation, based on the optimized geometries for each complex obtained as described in the text (for complex 2, the structure optimized using implicit CPCM acetonitrile solvation was used). Acetonitrile solvation (CPCM) was used during the TD calculation on complex 2; for the other complexes, no solvation was used. The B3LYP functional was used with def2tzvp on Fe and def2svp on all other atoms. Spectral simulations were generated using GaussSum ${ }^{16}$ and natural transition orbitals (NTOs) were generated using MultiWFN. ${ }^{17}$
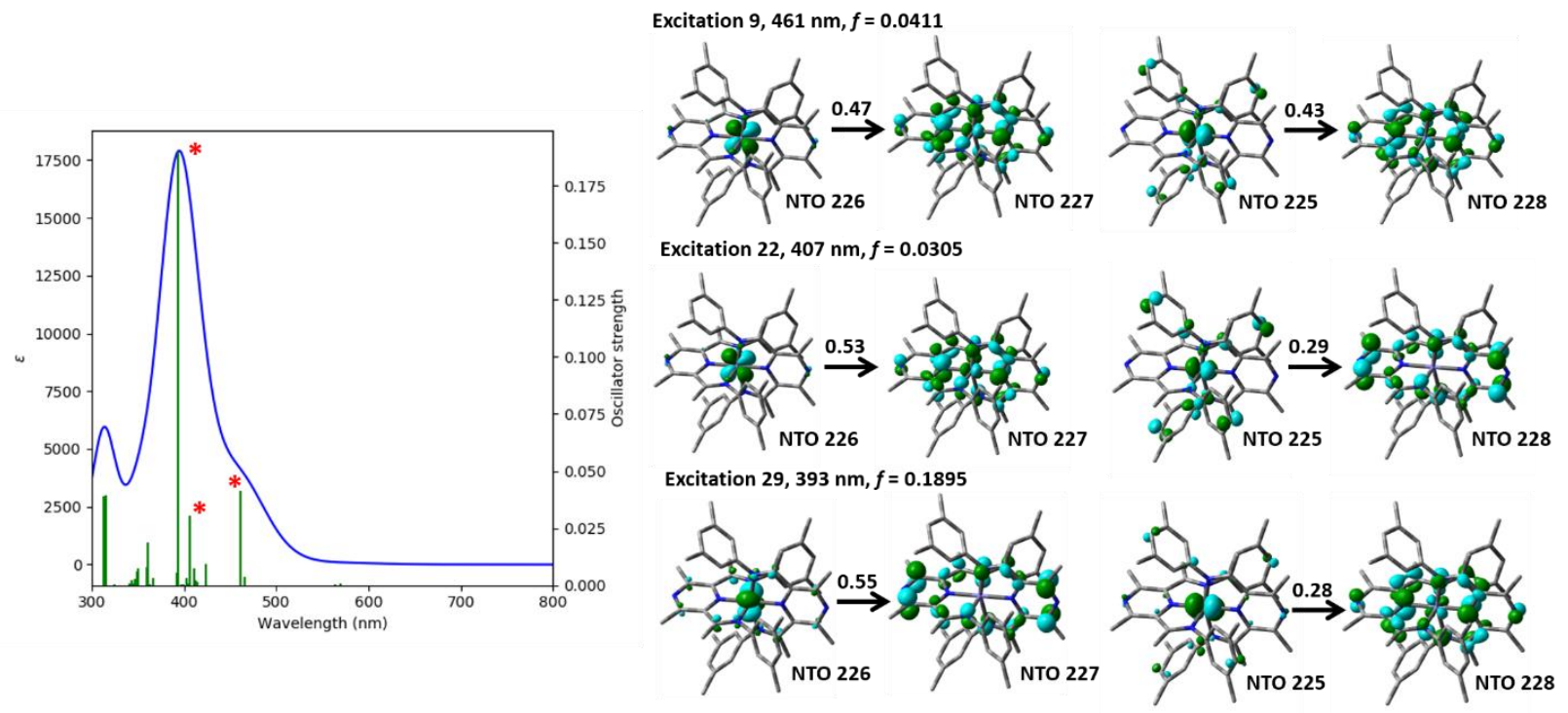

Figure S29. (Left) The first 60 excitations calculated for complex 1, $\mathrm{L}_{2} \mathrm{Fe}^{2+}$, convoluted with Gaussian functions; the three starred excitations below $350 \mathrm{~nm}$ in energy with oscillator strengths greater than 0.01 are shown. (Right) NTOs for the starred excitations. Numbers above the arrows are the relative contribution of each transition to the excitation. The three excitations shown are all primarily metal-to-ligand charge-transfer (MLCT) in character involving transitions from the iron $t_{2 g}$-like orbital set. The excitations are all singlet excitations $\left\langle\mathrm{S}^{2}\right\rangle=0$. 

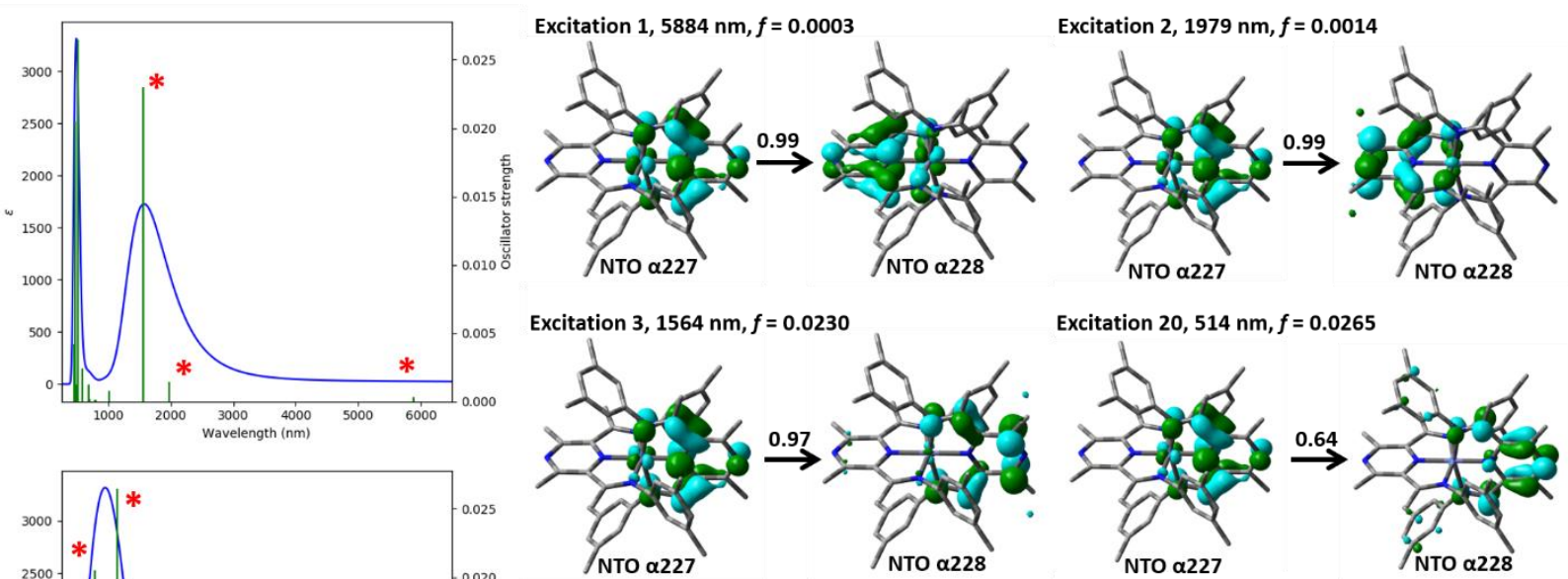

Excitation 20, $514 \mathrm{~nm}, f=0.0265$
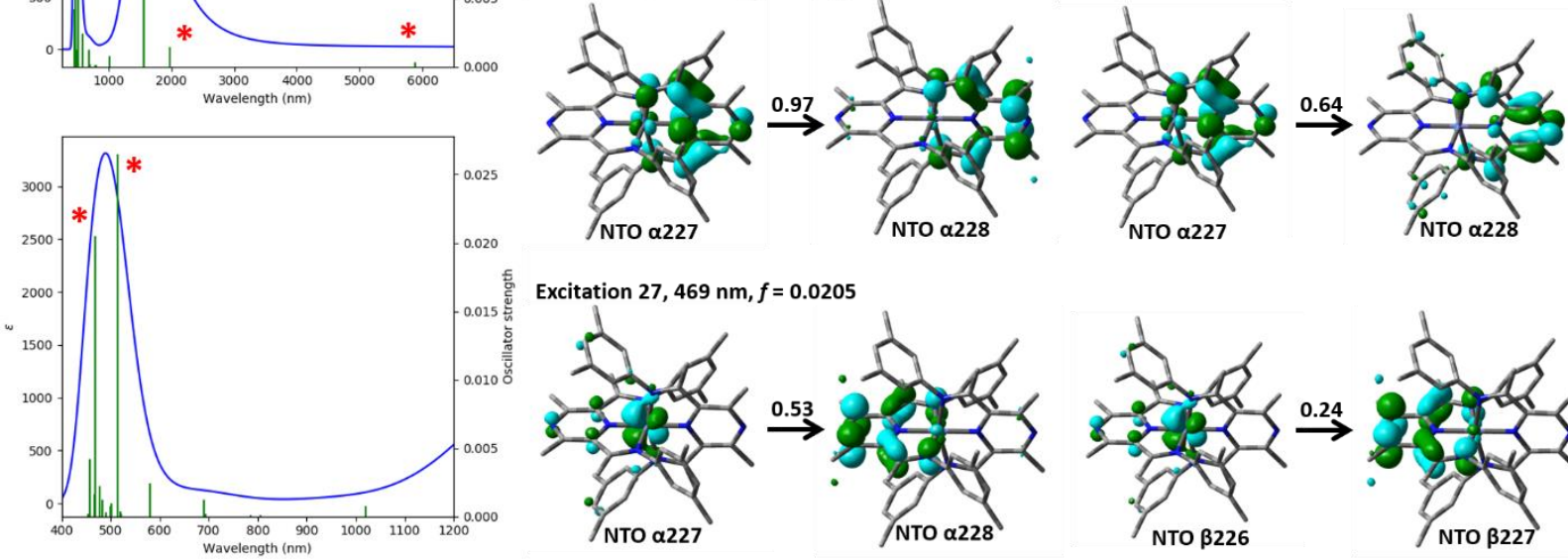

Figure S30. (Left) The first 30 excitations calculated for complex 2, $\mathrm{L}_{2} \mathrm{Fe}^{+}$, convoluted with Gaussian functions; full range (top) and visible region (bottom). (Right) NTOs for the starred excitations. Numbers above the arrows are the relative contribution of each transition to the excitation. The first two low-energy excitations are interligand (IVCT) transitions. The next two transitions are intraligand $\pi-\pi^{*}$ transitions in the $\alpha$ manifold involving the ligand anion radical, similar in character to those observed for complex $\mathbf{3}$ (see below), but localized on the one reduced ligand. The strong peak at $469 \mathrm{~nm}$ is an MLCT transition similar in character to the transitions shown above for complex 1 . 

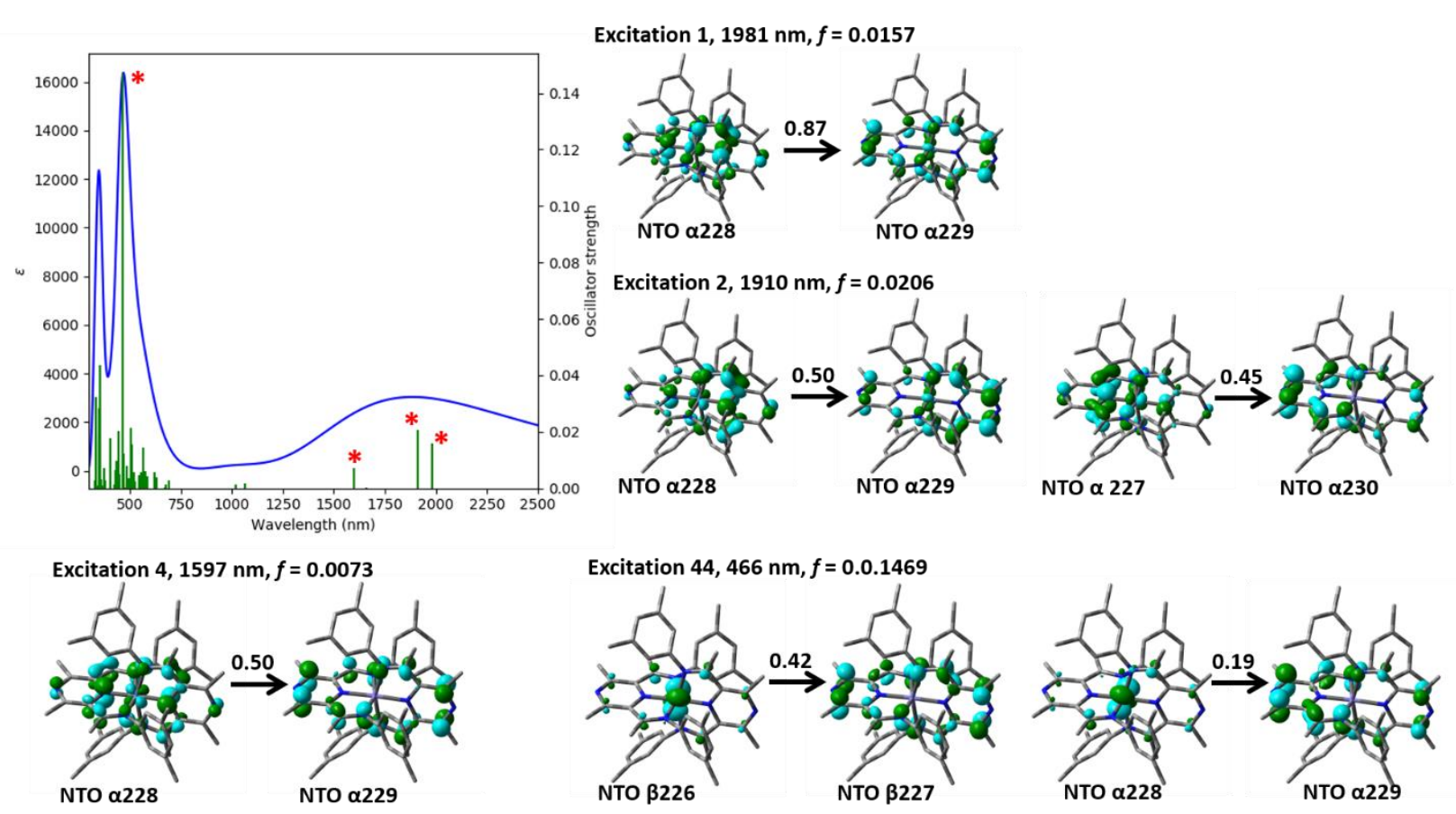

Figure S31. (Left) The first 60 excitations calculated for complex 3, $\mathrm{L}_{2} \mathrm{Fe}$, convoluted with Gaussian functions; selected excitations are shown in detail. (Right) NTOs for the starred excitations. Numbers above the arrows are the relative contribution of each transition to the excitation. The low-energy near-IR excitations $(1,2$, and 4$)$ are identified as primarily $\pi-\pi^{*}$ transitions in the $\alpha$ manifold involving the ligand anion radicals; the strong peak at $466 \mathrm{~nm}$ is an MLCT transition, primarily in the $\beta$ manifold, similar in character to the transitions shown above for complex 1.

\section{Supplemental References}

1. Regenauer, N. I.; Settele, S.; Bill, E.; Wadepohl, H.; Roşca, D.-A., Bis(imino)pyrazineSupported Iron Complexes: Ligand-Based Redox Chemistry, Dearomatization, and Reversible CC Bond Formation. Inorg. Chem. 2020, 59, 2604-2612.

2. Kilner, C. A.; Halcrow, M. A., An iron(II) complex of 2,6-di(pyrazol-1-yl)pyrazine that crystallises in three forms, two of which exhibit an unusual angular Jahn-Teller distortion. Polyhedron 2006, 25, 235-240.

3. Money, V. A.; Evans, I. R.; Elhaik, J.; Halcrow, M. A.; Howard, J. A. K., An x-ray powder diffraction study of the spin-crossover transition and structure of bis(2,6-dipyrazol-1ylpyrazine)iron(II) perchlorate. Acta Cryst. B 2004, 60, 41-45.

4. Elhaïk, J.; Money, V. A.; Barrett, S. A.; Kilner, C. A.; Evans, I. R.; Halcrow, M. A., The spin-states and spin-crossover behaviour of iron(II) complexes of 2,6-dipyrazol-1-ylpyrazine derivatives. Dalton Trans. 2003, 2003, 2053-2060.

5. Vallina, A. T.; Stoeckli-Evans, H.; Neels, A.; Ensling, J.; Decurtins, S., Iron-promoted nucleophilic additions to diimine-type ligands: A synthetic and structural study. Inorg. Chem. 2003, 42, 3374-3382. 
6. Campos-Fernandez, C. S.; Smucker, B. W.; Clerac, R.; Dunbar, K. R., Reactivity studies of 2,3,5,6-tetra(2-pyridyl) pyrazine (tppz) with first-row transition metal ions. Isr. J. Chem. 2002, 41, 207-218.

7. Toma, L. M.; Armentano, D.; De Munno, G.; Sletten, J.; Lloret, F.; Julve, M., 2,3,5,6Tetrakis(2-pyridyl)pyrazine (tppz)-containing iron(II) complexes: Synthesis and crystal structures. Polyhedron 2007, 26, 5263-5270.

8. Hofmann, A. J.; Jandl, C.; Hess, C. R., Structural differences and redox properties of unsymmetric diiron PDIxCy complexes. Eur. J. Inorg. Chem. 2020, 2020, 499-505.

9. Peng, D.; Zhang, Y.; Du, X.; Zhang, L.; Leng, X.; Walter, M. D.; Huang, Z., Phosphiniteiminopyridine iron catalysts for chemoselective alkene hydrosilylation. J. Am. Chem. Soc. 2013, 135, 19154-19166.

10. Gao, R.; Li, Y.; Wang, F.; Sun, W.-H.; Bochmann, M., 2-Benzoxazolyl-6-[1(arylimino)ethyl]pyridyliron(II) chlorides as ethylene oligomerization catalysts. Eur. J. Inorg. Chem. 2009, 2009, 4149-4156.

11. Cheung, P. M.; Burns, K. T.; Kwon, Y. M.; Deshaye, M. Y.; Aguayo, K. J.; Oswald, V. F.; Seda, T.; Zakharov, L. N.; Kowalczyk, T.; Gilbertson, J. D., Hemilabile Proton Relays and Redox Activity Lead to $\{\text { FeNO }\}^{x}$ and Significant Rate Enhancements in $\mathrm{NO}_{2}{ }^{-}$Reduction. J. Am. Chem. Soc. 2018, 140, 17040-17050.

12. Archer, A. M.; Bouwkamp, M. W.; Cortez, M.-P.; Lobkovsky, E.; Chirik, P. J., Arene coordination in bis(imino)pyridine iron complexes: Identification of catalyst deactivation pathways in iron-catalyzed hydrogenation and hydrosilation. Organometallics 2006, 25, 42694278.

13. Bruker, APEX3, SAINT and SADABS. Bruker AXS Inc.,: Madison, Wisconsin, USA., 2015. 14. Sheldrick, G. M., SHELXT - Integrated space-group and crystal-structure determination. Acta Cryst. A 2015, 71, 3-8.

15. Sheldrick, G. M., A short history of SHELX. Acta Cryst. A 2008, 64 (1), 112-122.

16. O'boyle, N. M.; Tenderholt, A. L.; Langner, K. M., cclib: A library for packageindependent computational chemistry algorithms. J. Comp. Chem. 2008, 29, 839-845. 17. Lu, T.; Chen, F., Multiwfn: A multifunctional wavefunction analyzer. J. Comp. Chem. 2012, 33, 580-592. 